\title{
Hidden Lineage Complexity of Glycan-Dependent HIV-1 Broadly Neutralizing Antibodies Uncovered by Digital Panning and Native-Like gp140 Trimer
}

OPEN ACCESS

Edited by:

Prabakaran Ponraj,

Intrexon, United States

Reviewed by:

Sanjay Kumar Phogat,

Sanofi Pasteur, United States

Bin Su,

Beijing You'an Hospital and Capital

Medical University, China

Neil S Greenspan,

Case Western Reserve University,

United States

*Correspondence:

Linling $\mathrm{He}$

linling@scripps.edu;

Jiang Zhu

jiang@scripps.edu

Specialty section:

This article was submitted to B Cell Biology,

a section of the journal

Frontiers in Immunology

Received: 23 June 2017 Accepted: 08 August 2017 Published: 24 August 2017

Citation:

He L, Lin X, de Val N, SayeFrancisco KL, Mann CJ, Augst R, Morris $C D$, Azadnia P, Zhou B, Sok D, Ozorowski G, Ward AB, Burton DR and Zhu J (2017) Hidden Lineage Complexity of Glycan-Dependent HIV-1 Broadly Neutralizing Antibodies Uncovered by Digital Panning and Native-Like gp140 Trimer.

Front. Immunol. 8:1025. doi: 10.3389/fimmu.2017.01025
Linling He ${ }^{1 *}$, Xiaohe Lin ${ }^{1}$, Natalia de Val ${ }^{2,3,4}$, Karen L. Saye-Francisco ${ }^{1,4}$, Colin J. Mann ${ }^{1}$, Ryan Augst ${ }^{1}$, Charles D. Morris ${ }^{1}$, Parisa Azadnia', Bin Zhou ${ }^{5}$, Devin Sok ${ }^{1,3,4}$, Gabriel Ozorowski ${ }^{2,3,4}$, Andrew B. Ward ${ }^{2,3,4}$, Dennis R. Burton ${ }^{1,3,4,6}$ and Jiang Zhu ${ }^{1,2,4 *}$

${ }^{1}$ Department of Immunology and Microbial Science, The Scripps Research Institute, La Jolla, CA, United States, ${ }^{2}$ Department of Integrative Structural and Computational Biology, The Scripps Research Institute, La Jolla, CA, United States, ${ }^{3}$ International AIDS Vaccine Initiative Neutralizing Antibody Center and the Collaboration for AIDS Vaccine Discovery, The Scripps Research Institute, La Jolla, CA, United States, ${ }^{4}$ Scripps Center for HIVIAIDS Vaccine Immunology and Immunogen Discovery, The Scripps Research Institute, La Jolla, CA, United States, ${ }^{5}$ Department of Chemistry, The Scripps Research Institute, La Jolla, CA, United States, ${ }^{6}$ Ragon Institute of Massachusetts General Hospital, Massachusetts Institute of Technology, Cambridge, MA, United States

Germline precursors and intermediates of broadly neutralizing antibodies (bNAbs) are essential to the understanding of humoral response to HIV-1 infection and B-cell lineage vaccine design. Using a native-like gp140 trimer probe, we examined antibody libraries constructed from donor-17, the source of glycan-dependent PGT121-class bNAbs recognizing the N332 supersite on the HIV-1 envelope glycoprotein. To facilitate this analysis, a digital panning method was devised that combines biopanning of phage-displayed antibody libraries, 900 bp long-read next-generation sequencing, and heavy/light $(\mathrm{H} / \mathrm{L})$-paired antibodyomics. In addition to single-chain variable fragments resembling the wild-type bNAbs, digital panning identified variants of PGT124 (a member of the PGT121 class) with a unique insertion in the heavy chain complementarity-determining region 1 , as well as intermediates of PGT124 exhibiting notable affinity for the native-like trimer and broad HIV-1 neutralization. In a competition assay, these bNAb intermediates could effectively compete with mouse sera induced by a scaffolded BG505 gp140.681 trimer for the N332 supersite. Our study thus reveals previously unrecognized lineage complexity of the PGT121-class bNAbs and provides an array of library-derived bNAb intermediates for evaluation of immunogens containing the N332 supersite. Digital panning may prove to be a valuable tool in future studies of bNAb diversity and lineage development.

Keywords: antibody phage display, B-cell lineage development, broadly neutralizing antibodies, HIV-1 vaccine design, native-like trimer, next-generation sequencing

\section{INTRODUCTION}

Broadly neutralizing antibodies (bNAbs) isolated from a small fraction of infected individuals have provided valuable insights into the humoral response against HIV-1 (1-4). It has been proposed that bNAbs with structurally defined antigen interactions can be used as templates for designing immunogens capable of eliciting similar antibody responses upon vaccination (5-7). Considering the 
extensive somatic hypermutation (SHM) and unusual sequence features of bNAbs, such as long complementarity-determining region (CDR) loops, an in-depth understanding of their ontogeny is imperative to designing sequential immunogens for guided antibody maturation (7). To this end, next-generation sequencing (NGS) has been utilized to explore details of the antibody repertoire and lineage development for bNAbs of vaccine interest (8-19). However, with the exception of a few cases $(16,17$, 19-22), studies regarding early bNAb development continue to be restrained by limited sample availability and low frequency of lineage intermediates in memory B cells. Nonetheless, germlinereverted precursors and inferred lineage intermediates have been derived for several bNAbs targeting the CD4-binding site (CD4bs) and the N332 supersite near the base of variable loop 3 (V3) to facilitate immunogen design and in vivo evaluation (18, 23-31).

The HIV-1 envelope glycoprotein (Env) is covered with a dense layer of glycans. While this "glycan shield" poses barriers for bNAbs to access epitopes such as the CD4bs (17), it harbors key neutralizing antibody targets $(32,33)$ including the trimeric apex and the N332 supersite, of which the latter is a high-mannose patch centered around the N332 glycan $(34,35)$. The N332-dependent bNAb classes represented by PGT121, PGT128, and PGT135 have been extensively studied, showing an inherent promiscuity of the N332 supersite (36-42). NGS has revealed sequence diversity within the PGT121 and PGT135 families $(9,15,18)$, with putative intermediates inferred for the former by phylogenetic analysis (18). The PGT121 class consists of bNAbs PGT121-123, PGT124/10-1074 (36), and PGT133-134, with PGT121 and 10-1074 demonstrating therapeutic potential in macaques and humans, respectively $(43,44)$. Structures of the PGT121-class bNAbs and their intermediates in complex with a modified gp120 core and BG505 SOSIP.664 gp140 trimer have placed PGT124 (and 10-1074 (36)) on a distinct evolutionary branch mainly focusing on the N332 glycan, whereas PGT121123 recruited multiple glycans during lineage maturation (41, 42, 45). Recently, Steichen et al. designed a series of SOSIP.664 trimers with optimized binding to the PGT121 precursor and inferred intermediates (31), which induced bNAb-like responses in immunoglobulin (Ig) knock-in mice (23). This study provided a proof of concept for the B-cell lineage vaccine design targeting the N332 supersite (7). Furthermore, a ferritin nanoparticle displaying 24 copies of an N332 epitope-scaffold was reported to elicit a consistent $\mathrm{N} 332$-specific antibody response in $\mathrm{BALB} / \mathrm{c}$ mice cross-reactive with a native-like trimer, which itself failed to induce such antibody response in immunization (46). Interestingly, once the membrane-proximal external region (MPER) and a C-terminal scaffold were included in this trimer construct, a robust antibody response to the apex was observed, indicating enhanced immune recognition of the glycan shield (46). Notwithstanding, several critical issues remain elusive in vaccine design targeting the N332 supersite: (i) whether native precursors and intermediates of the PGT121 class can be found within the donor repertoire; (ii) the minimal level of SHM required for the PGT121-class bNAbs to recognize an unmutated trimer; and (iii) whether a trimer immunogen with an intact glycan shield is capable of eliciting an N332-specific antibody response in animal immunization. A careful assessment of these issues is pertinent to future vaccine design efforts targeting the N332 glycan supersite.

In this study, we examined samples from donor-17, the source of PGT121-class bNAbs, to search for bNAb precursors and lineage intermediates. To facilitate this analysis, we developed a digital panning method by combining 900 bp NGS and H/L-paired antibodyomics with phage display of single-chain variable fragments (scFvs) to probe the donor repertoire. A biotinylated Avi-tagged BG505 gp140 trimer containing an optimized heptad repeat 1 (HR1) bend (47) was utilized as antigenic bait for antibody library screening. Although scFvs identified from this library may not possess authentic heavy and light chains, they nonetheless provide a glimpse into Env recognition by the diverse antibody repertoire that gave rise to the PGT121 lineage. Digital panning of a diverse scFv library identified bNAb-like clones with increased affinity for the native-like trimer, and PGT124 variants with a unique 2-aa insertion in the heavy chain complementarity-determining region 1 (HCDR1) loop. Similar sequences were also found in the donor antibody repertoire by deep sequencing, albeit with low frequency. A focused scFv library was then constructed using the PGT121 class-specific primers and subjected to digital panning against the trimer probe, revealing heavy and light chain (HC and LC) intermediates of the PGT121 class that differed notably from the inferred sequences (18). All library-derived antibody clones were assessed for antigen binding and HIV-1 neutralization. The utility of selected $\mathrm{bNAb}$ intermediates was further demonstrated using mouse sera from a previous trimer immunization (46). Serum analysis indicated that a scaffolded BG505 gp140.681 trimer induced consistent antibody responses to the apex and the N332 supersite, the latter of which could be blocked effectively by mature PGT124 and partially by a near-germline $\mathrm{HC}$ intermediate. Collectively, our study uncovers previously unrecognized lineage complexity of the PGT121-class bNAbs and presents a set of functional intermediates potentially valuable for the rational design and evaluation of HIV-1 immunogens containing the N332 supersite.

\section{MATERIALS AND METHODS}

\section{Human Specimen}

Peripheral blood mononuclear cells (PBMCs) from an HIV-1 infected donor (donor-17) (34) of the Protocol G cohort were used for scFv library construction and antibody repertoire sequencing.

\section{HIV-1 Panning Antigens}

The Avi-tagged BG505 gp140 trimer containing a redesigned HR1 bend (47) and the clade-C (ZM109) V1V2 nanoparticle (48) were transiently expressed in HEK293 F cells and in $N$-acetylglucosaminyltransferase I-negative $\left(\mathrm{GnTI}^{-/-}\right)$HEK293 S cells (Life Technologies), respectively. In brief, HEK293 F/S cells were thawed and incubated with FreeStyle ${ }^{\mathrm{TM}} 293$ Expression Medium (Life Technologies) in a Shaker incubator at $37^{\circ} \mathrm{C}$, with $120 \mathrm{rpm}$ and $8 \% \mathrm{CO}_{2}$. When cells reached a density of $2.0 \times 10^{6} /$ $\mathrm{ml}$, expression medium was added to reduce cell density to $1.0 \times 10^{6} / \mathrm{ml}$ for transfection with polyethylenimine (PEI-MAX) (Polysciences). For 1-1 transfection of the gp140 trimer, $800 \mu \mathrm{g}$ of plasmid, $300 \mu \mathrm{g}$ of furin plasmid, and $300 \mu \mathrm{g}$ of pAdVAntage were mixed in $25 \mathrm{ml}$ of Opti-MEM transfection medium (Life 
Technologies) and added to $25 \mathrm{ml}$ of Opti-MEM with $5 \mathrm{ml}$ of PEI-MAX $(1.0 \mathrm{mg} / \mathrm{ml})$. For $1-1$ transfection of the V1V2 nanoparticle, $900 \mu \mathrm{g}$ of plasmid was added to $25 \mathrm{ml}$ of Opti-MEM and then mixed with $5 \mathrm{ml}$ of PEI-MAX in $25 \mathrm{ml}$ of Opti-MEM. After incubation for $30 \mathrm{~min}$, the DNA-PEI-MAX complex was added to the cells. Culture supernatants were harvested 5 days after transfection, clarified by centrifugation at $1,800 \mathrm{rpm}$ for $22 \mathrm{~min}$, and filtered using $0.45 \mu \mathrm{m}$ filters (Millipore). A Galanthus nivalis lectin (GNL) column (Vector Labs) was used to extract HIV-1 antigens from the supernatants and eluted with PBS containing $500 \mathrm{mM}$ $\mathrm{NaCl}$ and $1 \mathrm{M}$ methyl- $\alpha$-D-mannopyranoside. For the Avi-tagged gp140 trimer, biotinylation was performed using the BirA biotinprotein ligase standard reaction kit (BirA-500) following the manufacturer's instructions (Avidity). The gp140 trimer and the V1V2 nanoparticle were then purified using size-exclusion chromatography (SEC) on a HiLoad 16/600 Superdex 200 PG column and a Superose $610 / 300$ GL column (GE Healthcare), respectively.

\section{Negative-Stain Electron Microscopy (EM)}

The biotinylated Avi-tagged BG505 gp140 trimer, termed gp140.664.R1-Avi-Biot, was analyzed by negative-stain EM using a previously published protocol (47). Briefly, images were acquired with a Tietz $4 \mathrm{k} \times 4 \mathrm{k}$ TemCam-F416 CMOS camera using a nominal defocus of $1,000 \mathrm{~nm}$ and the Leginon package (49) with an electron dose of $\sim 29 \mathrm{e}^{-} / \AA^{2}$. For image data processing, the Appion software package (50) was used to pick up particles and to make a stack. 2D classes were obtained using iterative multivariate statistical analysis (MSA)/multireference alignment (MRA) (51). To assess the quality of the trimers (native-like closed and open, or non-native), the reference-free $2 \mathrm{D}$ class averages were examined by eye using the same metrics as previously described (47). The 3D reconstruction of the gp140.664.R1-Avi-Biot trimer was obtained from the refinement of 17,783 particles using EMAN2 (52). The crystal structure of the BG505 SOSIP trimer (PDB ID: 4TVP) was fitted into the EM density and refined by using the UCSF Chimera "Fit in map" function (53).

\section{Biolayer Interferometry (BLI)}

Antibody-binding kinetics of the biotinylated gp140 trimer was assessed using an Octet RED96 instrument (fortéBio) as previously described (47). All assays were performed with agitation set to $1,000 \mathrm{rpm}$ in fortéBio $1 \times$ kinetic buffer. The final volume for all the solutions was $200 \mu \mathrm{l} /$ well. Assays were performed at $30^{\circ} \mathrm{C}$ in solid black 96-well plates (Geiger Bio-One). $5 \mu \mathrm{g} / \mathrm{ml}$ of protein in $1 \times$ kinetic buffer was used to load the HIV-1 antibody on the surface of anti-human Fc Capture Biosensors (AHC) for $300 \mathrm{s.}$ Typical capture levels were between 0.5 and $1 \mathrm{~nm}$ and variability within a row of eight tips did not exceed $0.1 \mathrm{~nm}$. A 60-s biosensor baseline step was applied prior to the analysis of the association of the antibody on the biosensor to the trimer in solution for $200 \mathrm{~s}$. A twofold concentration gradient of trimer starting at a maximum of $200 \mathrm{nM}$ was used in a titration series of six. The dissociation of the interaction was followed for $300 \mathrm{~s}$. Correction of baseline drift was performed by subtracting the mean value of shifts recorded for a sensor loaded with antibody but not incubated with trimer and for a sensor without antibody but incubated with trimer. Octet data were processed by fortéBio's data acquisition software v.8.1. For apex-directed bNAbs, experimental data were fitted with the binding equations describing a 1:1 interaction, whereas for other bNAbs, the binding equations describing a 2:1 interaction were utilized to obtain the optimal fitting. $K_{\mathrm{D}}$ values were determined using the estimated response at equilibrium for each trimer concentration rather than the $k_{\text {on }}$ and $k_{\text {off }}$ values.

\section{Antibody Phage Display}

The construction of scFv libraries was performed using a protocol modified from a previously described method (54). Briefly, total RNA was extracted from $\sim 10$ million PBMCs for single-stranded cDNA synthesis using the SuperScript ${ }^{\mathrm{TM}}$ III system (Life Technologies) with random hexamer and oligo(dT)12-18 primers. Antibody HC and LC variable regions were obtained from a primary polymerase chain reaction (PCR) with mixed $\mathrm{HuJ}$ reverse primers and separate $\mathrm{HuV}$ forward primers, including a set of forward primers designed to capture PGT121-class light chains containing framework region 1 (FR1) deletions (Table S1 in Supplementary Material). To generate HC-LC fragments, overlap PCR was performed in $25 \times 50 \mu \mathrm{l}$ reactions ( 10 cycles) with $50 \mathrm{ng}$ of gel-purified HC and $50 \mathrm{ng}$ of gel-purified LC ( $\lambda$ only or equal amounts of $\kappa$ and $\lambda$ LC) without primer. To obtain full-length scFv inserts, PCR was performed in $50 \times 50 \mu \mathrm{l}$ reactions ( 15 cycles) with SfiI-F and SfiI-R primers (Table S1 in Supplementary Material) using $100 \mathrm{ng}$ of gel-purified HC-LC as template. The resulting $s c F v$ inserts and the phagemid vector, $\mathrm{pAdL}^{\mathrm{TM}}-20 \mathrm{c}$ (Antibody Design Labs), were digested with SfiI and gel-purified. The scFv DNA (256 ng) was ligated into the phagemid vector (400 ng) using the T4 Ligase Kit (New England BioLabs) in $25 \times 40 \mu$ l reactions at $16^{\circ} \mathrm{C}$ overnight. Purified phagemids were electroporated into competent TG1 cells (Lucigen) with the MicroPulser ${ }^{\mathrm{TM}}$ system (Bio-Rad). Specifically, $1 \mu \mathrm{l}$ of phagemids and $25 \mu \mathrm{l}$ of competent cells were placed into a $0.1-\mathrm{cm}$ cuvette for electroporation using the pre-set program at $1.8 \mathrm{kV}$. The transformed bacteria were spread on 2 YT agar plates supplied with $100 \mu \mathrm{g} / \mathrm{ml}$ carbenicillin and $2 \%$ $(\mathrm{w} / \mathrm{v})$ glucose, which were incubated at $37^{\circ} \mathrm{C}$ overnight. Bacteria were then scraped from the plates for phage culture with the helper phage, CM13 (Antibody Design Labs), and biopanning. To facilitate NGS analysis, $3 \mathrm{ml}$ of the transformed bacteria was grown in $50 \mathrm{ml} 2 \mathrm{YT}$-Carb-Glu medium at $37^{\circ} \mathrm{C}$ for $2 \mathrm{~h}$, with the plasmids extracted using the Plasmid Midi Kit (Qiagen). Prior to biopanning, HIV-1 antigens were conjugated to magnetic beads following the manufacturer's instructions (Invitrogen), with Dynabeads ${ }^{\mathrm{TM}}$ M-280 Streptavidin beads used for biotinylated gp140 trimers and Dynabeads $^{\mathrm{TM}}$ M-270 Epoxy beads used for V1V2 nanoparticles. Four biopanning cycles were performed, with 6-8 wash steps in each cycle to remove phage that did not recognize the antigen. Plasmids were extracted from $3 \mathrm{ml}$ of the bacteria after each cycle for subsequent NGS analysis of scFv libraries.

\section{Next-Generation Sequencing}

Next-generation sequencing was performed on the Ion Torrent Personal Genome Machine (PGM) and S5 systems. The scFvcoding regions were amplified from the plasmid stock using PCR with fp1-SfiI-F and A-SfiI-[Barcode]-R primers (Table S1 in Supplementary Material). Of note, the forward primer (fp1-Sfil-F) contained a PGM full-length P1 (fP1) adaptor, whereas the 
reverse primer (A-SfiI-[Barcode]-R) contained a PGM A adaptor and an Ion Xpress ${ }^{\mathrm{TM}}$ barcode (Life Technologies) to differentiate each scFv library. A total of 25 PCR cycles were performed and the PCR products with an expected length of 800-900 bp were gel-purified (Qiagen). The procedure used for PGM sequencing has been described previously (20). Briefly, the libraries were quantitated using Qubit ${ }^{\circledR}$ 2.0 Fluorometer with Qubit ${ }^{\circledR}$ dsDNA HS Assay Kit. The dilution factor required for PGM template preparation was determined such that the final concentration was $50 \mathrm{pM}$. Template preparation was performed with the Ion PGM Template IA 500 Kit. Long-read sequencing was performed on the Ion Torrent PGM sequencer with the Ion PGM ${ }^{\mathrm{TM}} \mathrm{Hi}-\mathrm{Q}^{\mathrm{TM}}$ Sequencing Kit using an Ion 314 v2 chip for a total of 1,500 nucleotide flows. Raw sequencing data were processed without the $3^{\prime}$-end trimming in base calling to obtain full-length scFvs. The donor-17 HC library was generated using a $5^{\prime}$-RACE PCR protocol as previously described (17). Template preparation and (Ion 530) chip loading were performed on Ion Chef using the Ion 530 Ext Kit, followed by sequencing on the Ion S5 system with default settings. The raw NGS data can be found in the NCBI Sequence Read Archive with the accession number SRP105512.

\section{Bioinformatics Analysis of scFv Libraries}

The human Antibodyomics 1.0 pipeline $(8,15,17,55)$ has been adapted to analyze the $900 \mathrm{bp}$ sequencing data of scFv libraries. Following the housekeeping step (assigning a unique index to each $\mathrm{scFv}$ ) and light-chain germline gene assignment, each $\mathrm{scFv}$ sequence was divided into $\mathrm{HC}$ and $\mathrm{LC}$ by matching a 15 -aa $\left(\mathrm{G}_{4} \mathrm{~S}\right)_{3}$ linker connecting the two chains. The $\mathrm{HC}$ and LC datasets were then processed separately by the chain-specific antibodyomics pipelines. Finally, full-length $\mathrm{HC}$ and LC from the same scFv were re-matched according to their indices in the sequenced scFv library (scFv indices) and deposited into correlated HC and LC databases for more in-depth analysis.

The Antibodyomics 1.0 pipeline consists of the following five steps $(8,15,17)$ : (1) data reformatting and cleaning; (2) germline gene assignment; (3) sequencing error correction; (4) calculation of sequence identity to a set of known antibodies; and (5) determination of CDR3 sequences and variable domain boundaries. In this study, a number of changes have been made to improve the pipeline accuracy and efficiency. In step 2, the original pipeline assigned variable (V), diversity (D), and joining (J) genes sequentially for heavy chains, whereas the modified pipeline first assigned $\mathrm{V}$ and $\mathrm{J}$ genes and then determined an appropriate $\mathrm{D}$ gene for the region between the $\mathrm{V}$ and J gene segments. In step 3, BLASTn (56) was replaced with LALIGN (57) to generate pairwise local alignment, as BLASTn often outputs a partial alignment lacking the $\mathrm{N}$-terminus when sequencing errors occur in this region. By contrast, LALIGN can generate a more complete alignment with the assigned germline $\mathrm{V}$ gene, thus enabling error correction for the $\mathrm{N}$-terminus and proper translation to the protein sequence. Of note, two consecutive deletions or insertions separated by a single nucleotide may produce a merged gap in alignment due to a lower gap penalty. Such homopolymer errors can now be detected and corrected by the modified pipeline. In step 5, CLUSTALW2 (58) was replaced by MUSCLE (59) to generate multiple sequence alignment, with a reduction of computational time by threefold to fourfold. The modified pipeline (version 2.0) has been validated using the 454 sequencing data reported for donor-17 (18). The Antibodyomics 2.0 pipeline can be obtained upon request to the authors.

\section{H/L-Paired, CDR3-Based Clustering Analysis}

A novel method has been devised to determine non-redundant $\mathrm{scFv}$ clones and their frequencies within a converged phage library to facilitate antibody selection. This method is hierarchical, as it identifies the unique HCDR3 lineages first, and subsequently all the unique LCDR3 lineages associated with each HCDR3 lineage, resulting in a list of scFv clones each characterized by a distinct HCDR3-LCDR3 pair. In the first step, the unique HCDR3 lineages can be identified as follows: (1) HCDR3 loops are extracted from HC sequences and clustered into "groups" using CD-HIT (60) with an identity cutoff of $95 \%$ and a criterion of $\leq 1$ mismatch within the core alignment; (2) a consensus HCDR3 is derived for each HCDR3 group based on the multiple sequence alignment by MUSCLE; and (3) HCDR3 groups are merged into unique HCDR3 "lineages" by BLASTClust (56) with a sequence identity of $95 \%$ or greater covering $95 \%$ of the sequence length. For each HCDR3 lineage, a similar procedure can be applied to the matching LCs to determine the unique LCDR3 lineages. The resulting scFv clones will be ranked by their frequencies (the number of scFvs possessing a pair of unique HCDR3 and LCDR3), with the HC and LC sequence files pertaining to each $\mathrm{scFv}$ clone provided as the output. For large $\mathrm{scFv}$ clonal families (>500 members), which potentially represent highaffinity binders in the enriched library, an additional clustering analysis is performed to derive high-quality consensus sequences for antibody synthesis and functional validation. In brief, the $\mathrm{H} /$ LCDR3 sequences divisible by 3 will be clustered into groups using CD-HIT with an identity cutoff of $95 \%$, no mismatch within the core alignment and identical sequence lengths. The largest group is then manually inspected and subjected to the consensus calculation by MUSCLE using no more than 4,000 randomly selected sequences. For small scFv clonal families ( $\leq 500$ members), all sequences in the dataset are used to derive consensus HC and LC. The program used to perform H/L-paired, CDR3-based clustering analyses can be obtained upon request to the authors.

\section{Enzyme-Linked Immunosorbent Assay (ELISA)}

Each well of a Costar ${ }^{\mathrm{TM}}$ 96-well assay plate (Corning) was first coated with $50 \mu \mathrm{l}$ PBS containing $0.2 \mu$ g of the appropriate antigens. The plates were incubated overnight at $4^{\circ} \mathrm{C}$ and, then, washed five times with wash buffer containing PBS and $0.05 \%$ (v/v) Tween 20. Each well was then coated with $150 \mu$ of a blocking buffer consisting of PBS, $20 \mathrm{mg} / \mathrm{ml}$ blotting-grade blocker (Bio-Rad), and $5 \%(\mathrm{v} / \mathrm{v})$ FBS. The plates were incubated with the blocking buffer for $1 \mathrm{~h}$ at room temperature and, then, washed five times with wash buffer. Wild-type (WT) PGT121-class bNAbs and antibodies derived from the donor-17 scFv library were diluted in the blocking buffer to a maximum concentration of 1 or $10 \mu \mathrm{g} /$ $\mathrm{ml}$, followed by a 10 -fold dilution series. For each antibody dilution, a total of $50 \mu \mathrm{l}$ volume was added to the appropriate wells. 
Each plate was incubated for $1 \mathrm{~h}$ at room temperature and, then, washed five times with wash buffer. A 1:5,000 dilution of goat antihuman IgG antibody (Jackson ImmunoResearch Laboratories, Inc.) was then made in the wash buffer, with $50 \mu \mathrm{l}$ of this diluted secondary antibody added to each well. The plates were incubated with the secondary antibody for $1 \mathrm{~h}$ at room temperature and, then, washed five times with wash buffer. Finally, the wells were developed with $50 \mu \mathrm{l}$ of TMB (Life Sciences) for 3-5 min before stopping the reaction with $50 \mu \mathrm{l}$ of $2 \mathrm{~N}$ sulfuric acid. The resulting plate readouts were measured at a wavelength of $450 \mathrm{~nm}$.

\section{HIV-1 Neutralization}

Neutralization assays were performed on TZM-bl reporter cells using a panel of six tier- 2 isolates and two tier- 1 isolates. Neutralization curves were fit by a non-linear regression analysis using a 5-parameter hill slope equation. The 50\% inhibitory concentration $\left(\mathrm{IC}_{50}\right)$ is defined as the antibody concentration required for inhibiting HIV-1 infection by $50 \%$.

\section{Serum Binding and Competition ELISA}

Mouse antisera from a previous trimer immunization were tested against a native-like BG505 gp140 trimer (gp140.664.R1), a V1V2 nanoparticle (V1V2-FR), and an N332 nanoparticle (1GUT_A_ES-FR) by ELISA following a previously described protocol (46). Competition ELISA was performed to measure the binding of mouse antisera elicited by a scaffolded gp140 trimer to an N332 nanoparticle in the presence of WT bNAbs or native intermediates (NINs) derived from a focused donor-17 library. A slightly modified protocol was used. Briefly, plates were coated with purified N332 nanoparticles at $0.2 \mu \mathrm{g}$ per well and incubated overnight at $4^{\circ} \mathrm{C}$. After blocking, $10-50 \mu \mathrm{g} / \mathrm{ml}$ of a bNAb variant was added to the plates at $50 \mu \mathrm{l}$ per well for $1 \mathrm{~h}$ incubation. The mouse antisera were initially diluted by a factor of 5 in blocking buffer, followed by a 10 -fold dilution series. A $50 \mu \mathrm{l}$ volume of each dilution was then added to the wells without washing the plates. After incubation for $1 \mathrm{~h}$ and five washes, a 1:2,000 dilution of goat anti-mouse IgG antibody was added to each well at $50 \mu \mathrm{l}$ per well, followed by development with $50 \mu$ of TMB, stop with $50 \mu \mathrm{l}$ of $2 \mathrm{~N}$ sulfuric acid, and measurement at $450 \mathrm{~nm}$.

\section{RESULTS}

\section{A Native-Like gp140 Trimer Probe for Identification of Env-Specific Antibodies}

The HIV-1 Env spike, a trimer of gp120 and gp41 heterodimers, is the only target of neutralizing humoral immune response (61). Trimer-based HIV-1 vaccine design has long been hampered by the metastable nature of the Env. A cleaved, soluble BG505 SOSIP.664 gp140 trimer was recently developed that closely mimics the functional Env spike in the stable, prefusion conformation (62-68). The BG505 SOSIP.664 trimer has enabled high-resolution structural analysis of the Env spike in complex with many bNAbs by crystallography and EM $(42,69-75)$. Alternative trimer platforms have also been developed, including the native, flexibly linked trimer (76-78), the single-chain gp140 (sc-gp140) trimer (79), and the uncleaved, prefusion-optimized (UFO) trimer (47). In particular, the UFO design has demonstrated greater trimer yield and purity for diverse HIV-1 strains in comparison to the SOSIP design (47). Furthermore, a cleaved version of the UFO trimer has been displayed on various nanoparticles as multivalent immunogens (48).

Here, we designed a cleaved BG505 trimer probe for Envspecific antibody identification, which contains a redesigned HR1 bend-the basis of the UFO design (47) — and a C-terminal Avitag. This trimer probe was expressed transiently in HEK293 F cells with co-transfected furin as previously described (47). The secreted Env protein was purified using a GNL column followed by in vitro biotinylation and SEC on a Superdex 200 16/600 column. We first compared the SEC profiles of the tagged and untagged trimers based on the ultraviolet absorbance at $280 \mathrm{~nm}$ (Figure 1A). The biotinylated Avi-tagged trimer probe, termed gp140.664.R1-AviBiot, displayed high yield and purity on par with the untagged trimer. We then analyzed the trimer probe by negative-stain EM, which yielded a $3 \mathrm{D}$ reconstruction consistent with the previously reported structures of HR1-redesigned trimers and UFO trimers (Figure 1B; Figure S1 in Supplementary Material) (47). The unoccupied EM densities extending from the C-termini of the docked gp140 trimer model may correspond to the biotinylated Avi-tag. Using BLI, we assessed the antigenicity of the trimer probe against a panel of bNAbs and non-NAbs. Three bNAbs isolated from donor-17 (PGT121, 124, and 133) (34) were used to assess the recognition of the $\mathrm{N} 332$ supersite by the PGT121-class bNAbs and to establish a baseline for comparison with forthcoming antibodies identified from this donor (Figure 1C). Octet binding revealed similar kinetics for the trimer probe and the untagged trimer, showing fast on-rates and flat dissociation curves. Consistently, nearly identical kinetic profiles were observed for bNAbs targeting other conserved epitopes, including the apex recognized by PGDM1400 (80) and PG16 (35), the CD4bs by VRC01 (81), and the gp120-gp41 interface by PGT151 (82) and 35O22 (83) (Figure 1D). Five non-NAbs were utilized to assess the exposure of non-neutralizing epitopes on the Env surface (Figure 1E). While the trimer probe appeared to shield non-neutralizing epitopes to the same degree as the untagged parent trimer, both outperformed the SOSIP trimer (47).

Our results thus demonstrated that the addition of an Avi-tag and in vitro biotinylation had no adverse effect on trimer purity, structural integrity, or antigenicity. In recent studies, the BG505 SOSIP.664 trimer was used as a bait to isolate bNAbs from HIV-1 patient samples $(84,85)$. As one of the well-characterized trimer platforms $(86,87)$, the UFO trimer may provide an alternative probe to identify bNAbs of diverse specificities from antibody libraries or Env-specific B cells.

\section{Long-Read (900 bp) NGS and H/L-Paired Antibodyomics Enable Digital Panning}

Phage display $(88,89)$ has been widely used to produce monoclonal antibodies (mAbs) for research and therapeutic purposes (90-94). While the first HIV-1 bNAbs were isolated from phage libraries (95), single-cell approaches have contributed most of the new bNAbs (96). Nonetheless, both scFvs and fragment antigen binding regions can be displayed on the phage surface and subjected to a selection process known as biopanning. Although NGS has been used to estimate the diversity of antibody phage 


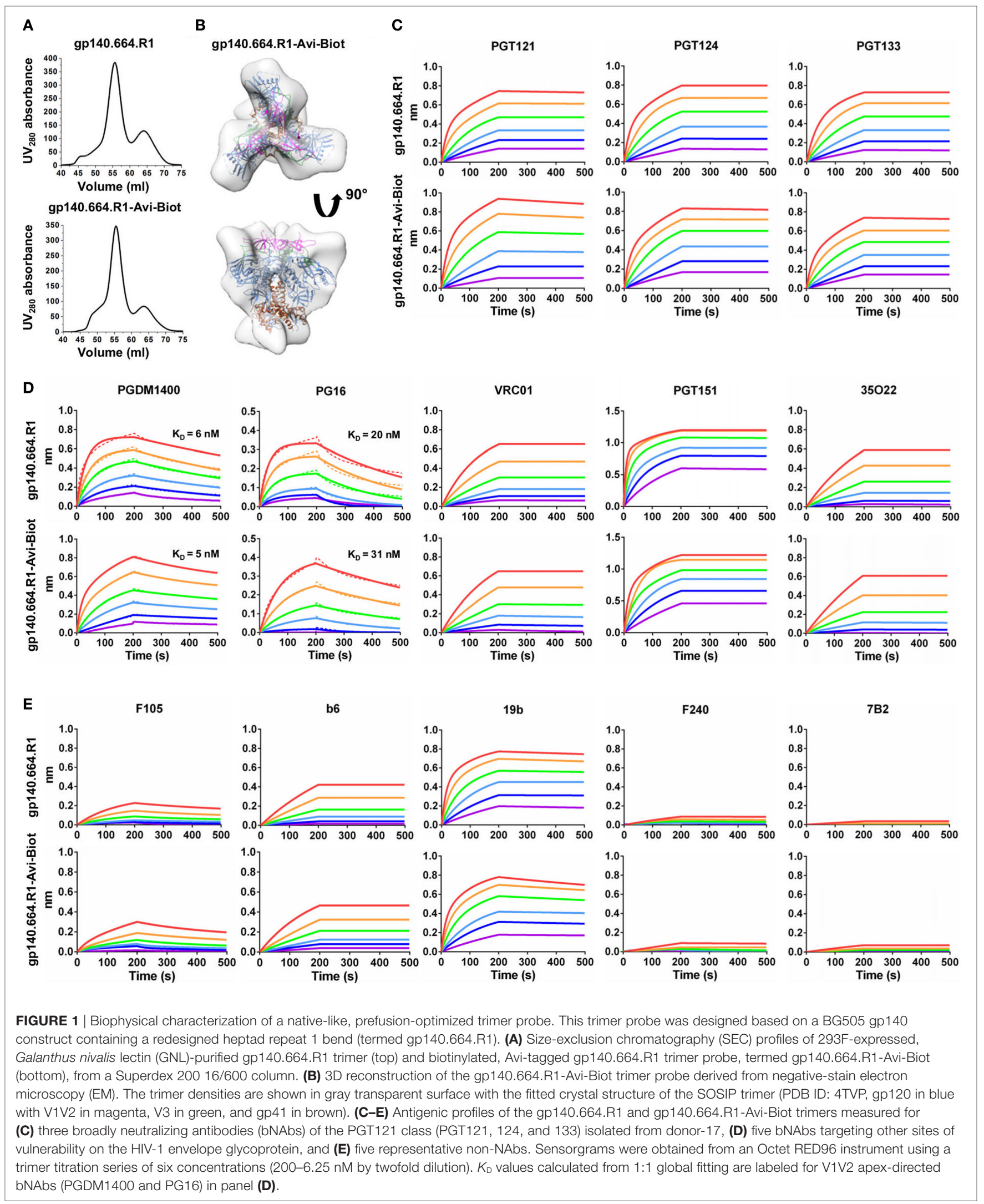


libraries $(97,98)$, its broader application has been restricted by the insufficient read length to cover both $\mathrm{HC}$ and LC in a scFv sequence $(>800 \mathrm{bp})$. It was recently proposed that once this technical barrier is overcome, NGS can be used to directly select functional mAbs from a scFv library $(99,100)$, rendering a quantitative method for antibody discovery.

Here, we addressed this technical challenge in a case study of the PGT121 lineage. We first characterized three types of antibody libraries constructed from PBMCs of donor-17 using gel electrophoresis (Figure 2A). Two antibody libraries were generated using different PCR methods-multiplex PCR (with gene-specific primers) and 5'-RACE PCR (with single 3 '-reverse primers) - and showed bands around 500 and $600 \mathrm{bp}$, respectively (15). By contrast, the $\mathrm{scFv}$ library generated using H/L-overlapping PCR and a large primer set (Table S1 in Supplementary Material), as well as four post-panning libraries, yielded distinctive bands around $900 \mathrm{bp}$. This comparison highlights the difficulty in sequencing full-length scFv libraries. Previously, we demonstrated an extended read length of 600-700 bp in the unbiased NGS analysis of antibody repertoires using the Ion Torrent PGM and a modified protocol with 1,200 nucleotide flows $(15,17,46,101)$. Can this NGS platform be adapted for sequencing scFvs? To test this possibility, we sequenced the donor-17 scFv libraries on the PGM using an Ion $314 \mathrm{v} 2$ chip and 1,500 nucleotide flows (Figure 2B). Markedly, over one million raw reads in the range of 750-950 bp were obtained for the scFv libraries, compared with 450 and $550 \mathrm{bp}$ for the mixed HC/LC libraries generated by two different PCR methods.

We then combined 900 bp long-read NGS and H/L-paired antibodyomics with scFv library panning into a coherent strategy, termed digital panning, for identification of functional mAbs (Figure 2C). By design, this method is both analytical and deterministic, as it can capture the full-length scFvs during the panning process and select representative $\mathrm{scFv}$ clones based on their frequency and antibody characteristics. The previously developed Antibodyomics pipeline (55) was modified to facilitate in silico analysis of the sequenced scFv libraries (Figure 2D). Briefly, each $\mathrm{scFv}$ is assigned a unique index and divided into $\mathrm{HC}$ and $\mathrm{LC}$ by matching a 15-aa linker between the two chains. Following the chain-specific pipeline processing, $\mathrm{HC}$ and LC from the same $s c F v$ are identified based on their shared $s c F v$ indices, resulting in correlated HC and LC databases. Of note, the method used in the Antibodyomics pipeline for indel error correction (9) has been modified to achieve greater accuracy, as demonstrated for the 454 sequencing data from donor-17 (18) (Figure S2 in Supplementary Material). The $\mathrm{scFv}$-derived $\mathrm{HC}$ and LC databases can then be analyzed in-depth using bioinformatics tools previously developed for repertoire profiling and lineage tracing $(8-12,15,17)$. Further implementation of an H/L-paired, CDR3-based clustering method allows determination of representative $s c F v$ clones and their respective frequencies for facilitating $\mathrm{mAb}$ selection.

\section{Digital Panning of a Diverse Donor-17 ScFv Library Identifies a New Lineage Variant}

As described earlier, a diverse donor-17 scFv library has been constructed using primers covering all $\mathrm{HC}$ and LC germline gene families (Table S1 in Supplementary Material) and screened against a native-like trimer probe (Figure 1). The pre-panning scFv library and four post-panning scFv libraries were pooled at a ratio of 3:1:1:1:1 for 900 bp deep sequencing on the PGM (Table S2A in Supplementary Material). After data processing with the H/L-paired Antibodyomics pipeline (Figure 2D), the scFv-derived HC and LC databases were analyzed to generate library profiles and to select Env-specific mAb clones.

Quantitative library profiles displayed a distinct pattern of antibody enrichment and a rapid convergence after two panning steps. In terms of germline gene usage (Figure 3A, column 1), the IgHV 4 and IgLV3 gene families accounted for $>96 \%$ of the library upon convergence, with a 6-12-fold increase in frequency with respect to the pre-panning library. Further analysis revealed the prevalence of IgHV4-59 ( 60\%) and IgHV4-61 ( 30\%) within the IgHV4 family and IgLV3-21 ( 96\%) within the IgLV3 family. In contrast, the $\kappa$ LCs exhibited a less discernible pattern of germline gene usage and a reduction to less than 100 sequences in the converged scFv library. A significant shift was observed in the SHM distribution (Figure 3A, column 2), with the average value increasing from $11 \%$ and $6 \%$ to $24 \%$ and $27 \%$ for $\mathrm{HC}$ and LC, respectively. Furthermore, nearly $90 \%$ of the HC sequences possessed long HCDR3 loops of 23-25 aa (Figure 3A, column 3 ). Overall, this $s c F v$ library appeared to have converged to the PGT121-class bNAbs, which are characterized by a specific germline gene usage (IgHV4-59 and IgLV3-21), high degree of SHM (HC: $19-22 \%$ and LC: $21-29 \%$ ), and a long HCDR loop (24 aa). A two-dimensional (2D) identity-divergence analysis was then performed to visualize the $\mathrm{scFv}$-derived $\mathrm{HC}$ and LC populations during the trimer panning process (Figure 3B). Indeed, the 2D plots revealed rapid enrichment of PGT124-like HCs and PGT124/PGT133-like LCs, which was further confirmed by the $\mathrm{H} / \mathrm{L}$-paired, CDR3-based clustering analysis (Figure 3C). While the most prevalent scFv family was characterized by PGT124like HCDR3 loops, $30 \%$ of HCs in this family were assigned to IgHV4-61 instead of IgHV4-59 due to a 2-aa insertion in HCDR1. Of note, the PGT133-like LCs appeared to be more prevalent than the PGT124-like LCs in the converged library, showing 50-fold difference in their frequencies. In addition, a small group of 45 scFvs possessed a 16-aa HCDR3 loop partially matching the C-terminal portion of the PGT124 HCDR3 sequence. Taken together, digital panning of a diverse donor-17 scFv library resulted in a panel of mAbs including predominant bNAb-like clones and mAbs of unknown specificities.

The six most prevalent mAbs $\left(\mathrm{Ab}_{\mathrm{d} 17}-1-6\right)$ in the $\mathrm{H} / \mathrm{L}$-paired clustering analysis (Figure 3C) were synthesized for functional validation (Table S3A in Supplementary Material). Antigen binding was initially assessed by ELISA against a native-like trimer (gp140.664.R1) (47), a gp120-ferritin (gp120-FR) nanoparticle (48), and an N332 nanoparticle (1GUT_A_ES-FR) (46), with a V1V2-ferritin nanoparticle (V1V2-FR) (48) included as a negative control. Three representative bNAbs for the PGT121 class demonstrated differential binding profiles: PGT124 bound to the trimer and the N332 nanoparticle with comparable EC50s, whereas PGT121 showed preferential binding to the gp140 trimer (Figure 4A). This finding is consistent with the reports that PGT121 engages multiple glycans on the Env $(41,42)$, which may not all 


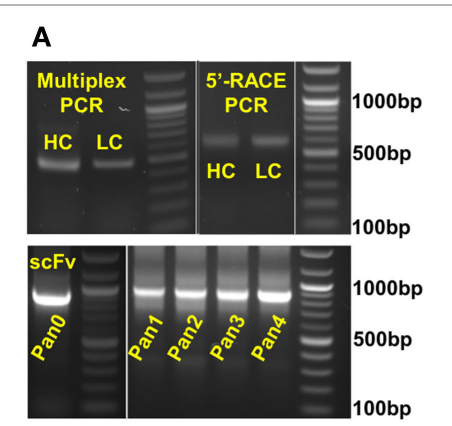

C

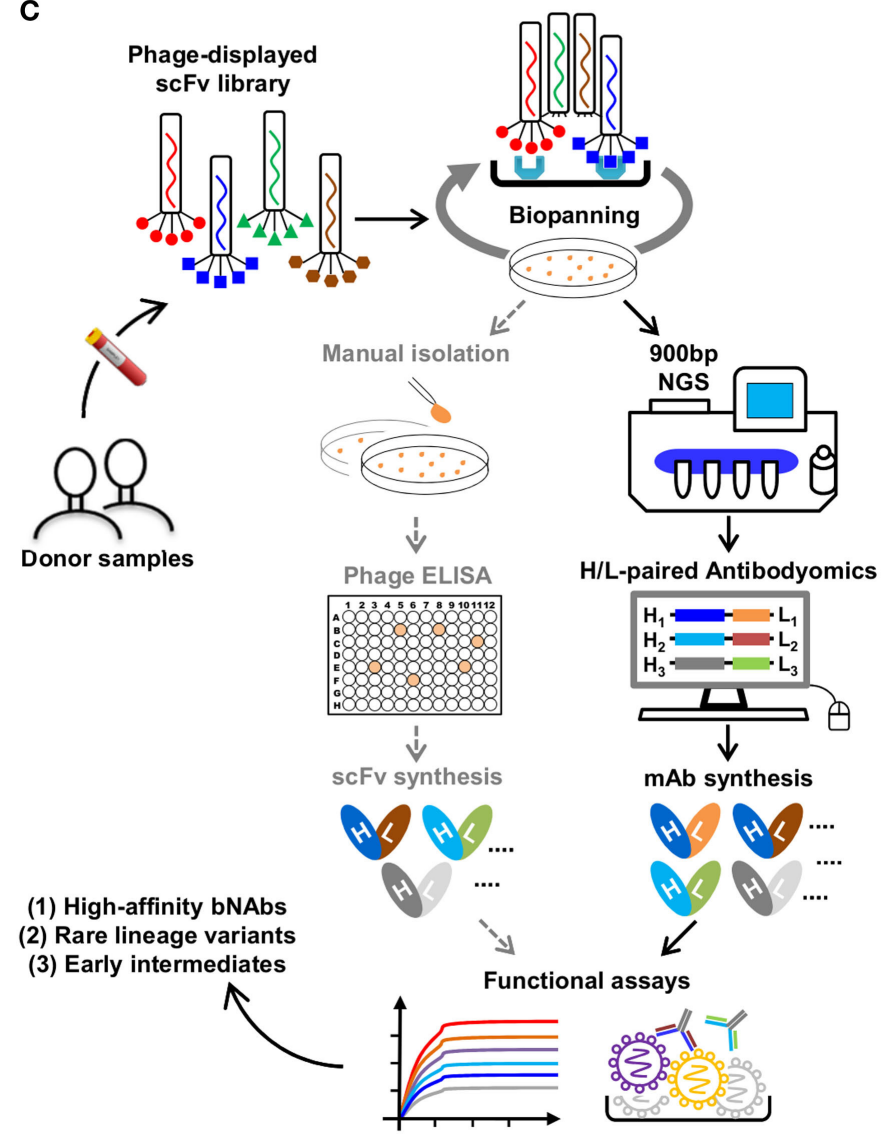

B

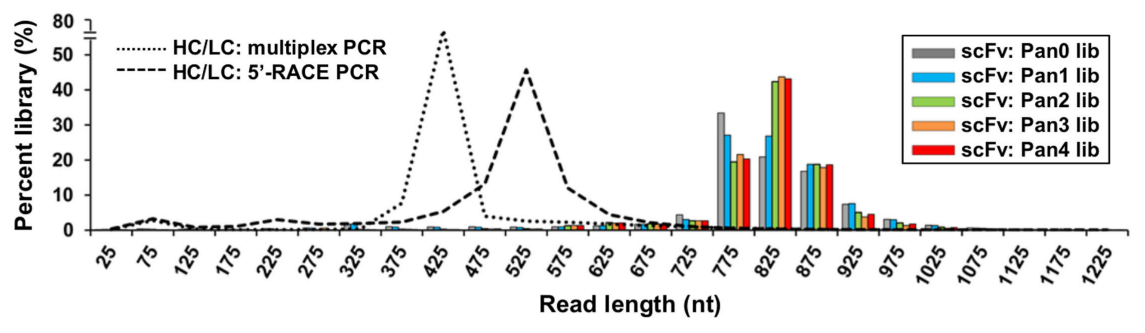

D

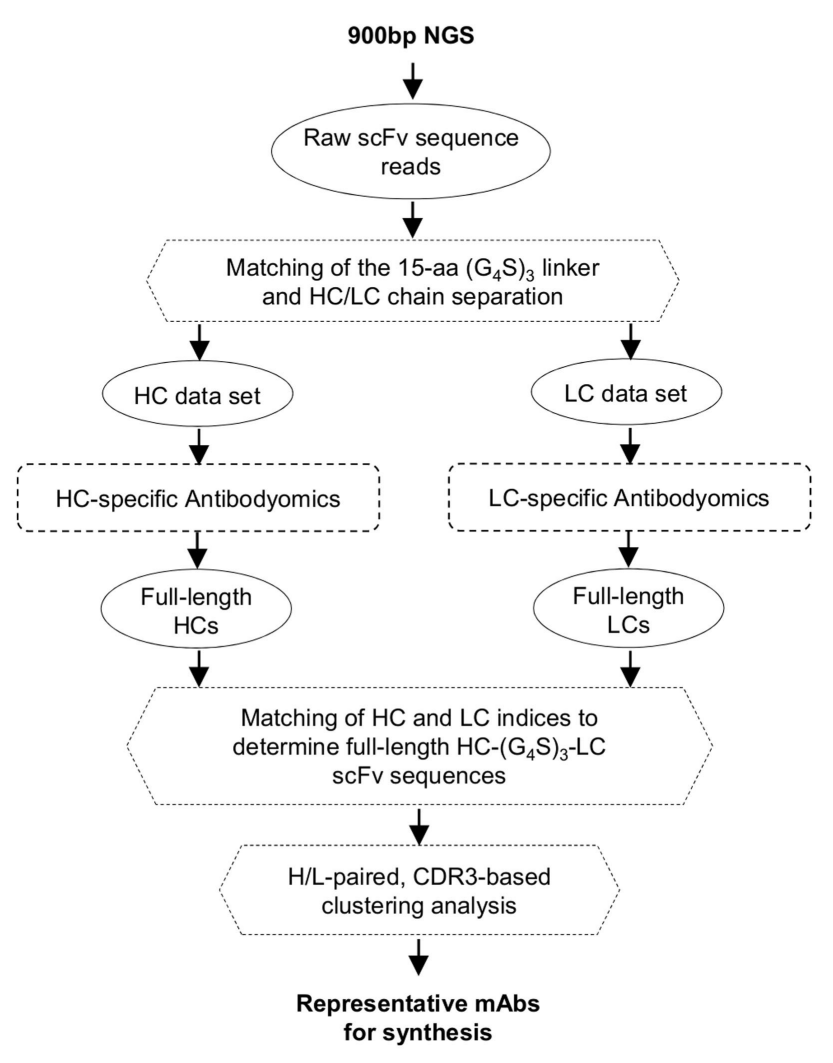

FIGURE 2 | Digital panning, a quantitative method for identification of functional antibodies. (A) Gel electrophoresis of three donor-17 antibody libraries generated from multiplex PCR using gene-specific primers, 5'-RACE PCR using single 3'-reverse primers, and H/L-overlapping PCR using gene-specific primers. For the purpose of formatting, the DNA gel has been rearranged with splicing (labeled with white lines). (B) Read length distributions of three donor-17 antibody libraries obtained from Personal Genome Machine (PGM) sequencing. Five single-chain variable fragment (scFv) libraries are plotted as histograms and color-coded according to their antigen panning steps: gray (Pan0), cyan (Pan1), green (Pan2), orange (Pan3), and red (Pan4). The two mixed HC/LC libraries generated from multiplex PCR and 5'-RACE PCR are shown as black dotted and dashed lines, respectively. (C) Schematic view of the digital panning method with the route of conventional panning included for comparison. Briefly, a scFv library is first constructed from donor peripheral blood mononuclear cells (PBMCs) and displayed on the phage surface for biopanning against an optimized antigen. The pre- and post-panning scFv libraries are sequenced on PGM to achieve 900 bp read length, with the sequenced full-length scFv libraries processed and analyzed by an $\mathrm{H} / \mathrm{L}$-paired antibodyomics pipeline. Representative scFv clones are selected for mAb synthesis and functional characterization. In HIV-1 bNAb studies, digital panning may identify high-affinity bNAb-like scFvs, rare bNAb lineage variants, and early intermediates. (D) Schematic view of an $\mathrm{H} / \mathrm{L}$-paired antibodyomics analysis.

be included in the scaffolded N332 supersite on the nanoparticle surface (46). Among the library-derived mAbs, the two PGT124like clones $\left(\mathrm{Ab}_{\mathrm{d} 17}-1\right.$ and $\left.\mathrm{Ab}_{\mathrm{d} 17}-2\right)$ appeared to be the best performers, binding to the N332-containing antigens with EC50 values $\sim 10$-fold lower than the WT bNAbs in addition to the reduced
V1V2 recognition. Among the other mAbs, $A b_{\mathrm{d} 17}-5$ and $A b_{d 17}-6$ bound the native-like trimer and the gp120 nanoparticle but not the N332 nanoparticle, whereas $A b_{\mathrm{d} 17}-3$ and $A b_{\mathrm{d} 17}-4$ displayed poor antigen binding. The "YYY-MDV" segment in the $A b_{\mathrm{d}_{17}}-6$ HCDR3, which partially matches to the PGT124 HCDR3, might 
be involved in Env binding, but detailed structural characterization would be required to confirm this hypothesis. When evaluated by BLI (Figure $4 \mathrm{~B}$ ), $\mathrm{Ab}_{\mathrm{d} 17}-1$ and $\mathrm{Ab}_{\mathrm{d} 17}-2$ exhibited PGT124-like trimer binding profiles in comparison with a weak binding signal observed for $A b_{d 17}-6$. HIV-1 neutralization was tested against a panel of six tier-2 viruses and two tier-1 viruses (Figure 4C). While $A b_{d 17}-1$ and $A b_{d 17}-2$ displayed the same neutralization breadth and potency as the WT bNAbs, Ab ${ }_{17}-6$ only neutralized

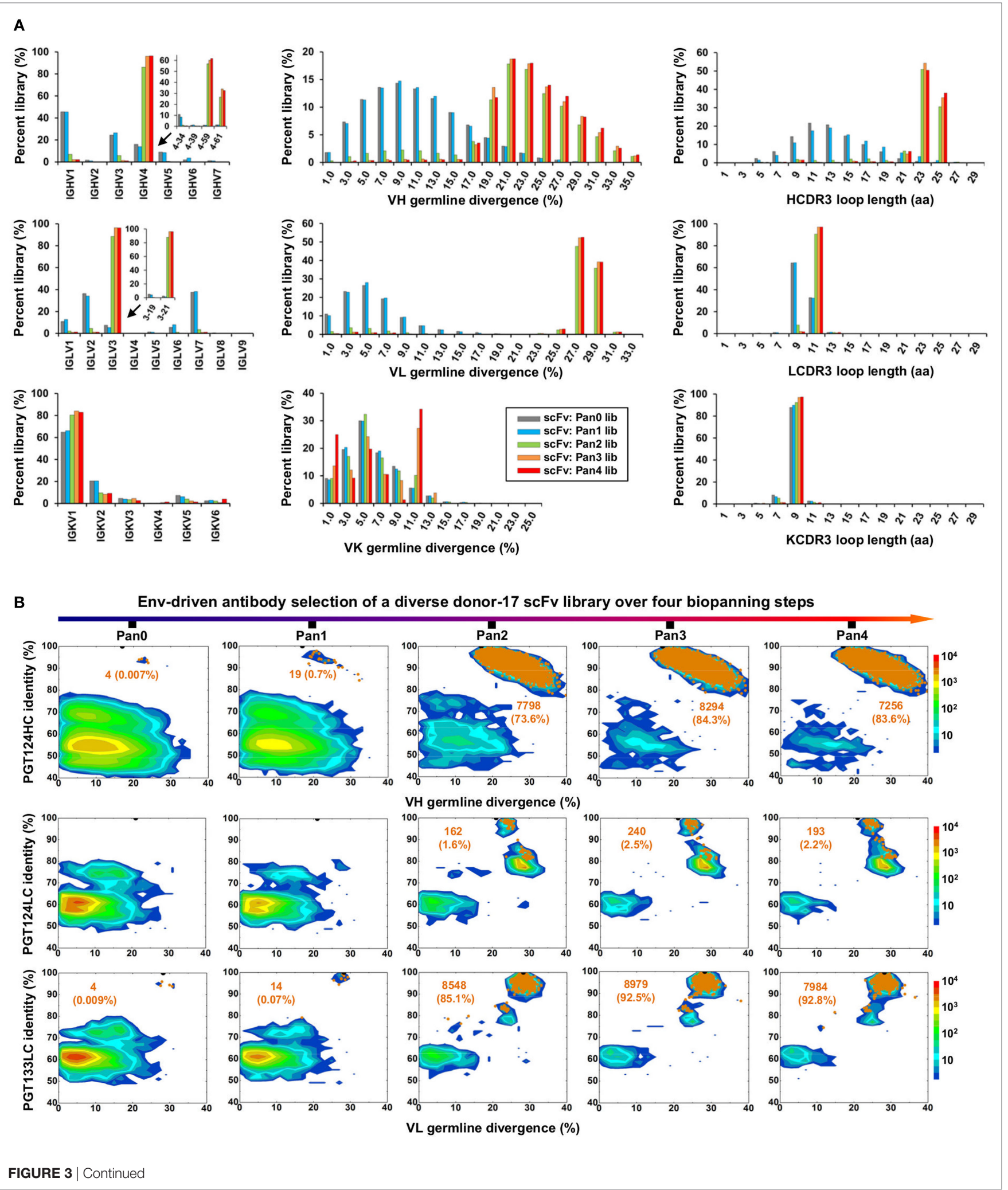


C

Representative scFv/mAb clones identified by H/L-paired, CDR3-based clustering analysis a

\begin{tabular}{|c|c|c|c|c|c|c|c|}
\hline Clone & $\mathrm{HC}$ name & VH identity & HCDR3 (aа) & Size & LC name & LCDR3 (aa) & Size \\
\hline$A b_{d 17}-1$ & T2P4H1 & $85.1 \%$ & & & T1P4L1 & HYWDSRSPISWI & 6,910 \\
\hline$A b_{d 17}-2$ & $\mathrm{~T} 2 \mathrm{P} 4 \mathrm{H} 2^{\mathrm{b}}$ & $84.3 \%$ & ARRGQRIYGVVSFGEFFYYYYMDV| & 7,277 & T1P4L2 & HMWDSRSGFSWS & 133 \\
\hline$A b_{d 17}-3$ & T2P4H1 & & & & T2P4L3 & QSYDSSLSGVV & 10 \\
\hline $\mathrm{Ab}_{\mathrm{d} 17}-4$ & T2P4H3 & $88.2 \%$ & DGGDPDTPWFIGAFDV & 31 & T2P4L3 & QSYDSSLSGVV & 24 \\
\hline$A b_{d 17}=5$ & T2P4H4 & $91.2 \%$ & ATAPWSH & 19 & T2P4L4 & LLYHSGAQPYVV & 15 \\
\hline $\mathrm{Ab}_{\mathrm{d} 17}-6$ & T2P4H5 & $93.2 \%$ & DGEWQIMNYYYKGMDV & 45 & T2P4K5 & QQSFTTPQT & 12 \\
\hline
\end{tabular}

a Listed items include scFv/mAb clone name, heavy chain (HC) name, VH germline gene identity (\%), HCDR3 sequence, HC cluster size, light chain (LC) name, LCDR3 sequence, and LC cluster size. The bracket indicates that $A b_{d 17}-1-3$ HCs share the same HCDR3 sequences, thus belonging to a single HCDR3 cluster.

b T2P4H2 shares the same HCDR3 sequence as WT PGT124 HC but contains a 2-aa mutation in the HCDR1 loop.

FIGURE 3 | Digital panning of a diverse donor-17 single-chain variable fragment (scFv) library against the native-like trimer. A diverse scFv library was constructed from donor-17 peripheral blood mononuclear cells (PBMCs) using a complete set of primers. (A) Distributions of germline gene usage (left), somatic hypermutation (middle), and CDR3 loop length (right) are plotted for the five scFv libraries obtained from the trimer panning process. Distributions of prevalent germline gene families within IgHV4 and IgLV3 (>1\%) are shown as insert images. Histograms are color-coded following the same scheme as in Figure 2B. (B) Identity-divergence analysis of the five donor-17 scFv libraries obtained from the trimer panning process. For each library, sequences are plotted as a function of sequence identity to PGT124 HC (top), PGT124 LC (middle), and PGT133 LC (bottom), and sequence divergence from germline genes. Color-coding indicates sequence density at a particular point on the 2D plot. Wild-type (WT) PGT124 and PGT133 are labeled on the 2D plots as black dots. Sequences with CDR3 identity of $95 \%$ or greater are shown as orange dots, with the number of sequences and library percentage labeled on the 2D plot for comparison. (C) Representative scFv clones identified from a diverse scFv library by H/L-paired, CDR3-based clustering analysis for mAb synthesis.

two tier-1 viruses, indicating the presence of diverse NAb lineages, including the PGT121 lineage, in the donor repertoire.

A key finding thus far in the analysis of a diverse donor-17 $s c F v$ library was $\mathrm{Ab}_{\mathrm{d} 17}-2$, which represented PGT124-like clones with a putative IgHV4-61 origin. Sequence alignment of $\mathrm{Ab}_{\mathrm{d} 17}-2$ with IgHV1-59, IgHV4-61, and PGT124 revealed a unique 2-aa insertion preceding the "YY" motif in the HCDR1 loop (Figure 4D). Is this insertion biologically relevant or merely an error that occurred in the scFv library construction? To answer this question, we prepared an HC-only library from the donor-17 PBMCs (that were used to construct scFv libraries) with a previously reported $5^{\prime}$-RACE PCR protocol $(15,17$, $46,101)$ and sequenced this library on the Ion S5 platform using an Ion 530 chip. S5 sequencing yielded over 12 million raw reads, which were processed by the Antibodyomics 2.0 pipeline, resulting in 7.8 million full-length HCs for repertoire profiling and identity-divergence analysis (Figures S3A,B in Supplementary Material). HC populations with a PGT124 identity of $85-100 \%$ and a germline divergence of $15-25 \%$ were observed on the $2 \mathrm{D}$ plot (Figure 4E). Among the 761 sequences with an HCDR3 identity of $90 \%$ or greater to PGT124, 11 were assigned to IgHV4-61 with HCDR1 insertions. Interestingly, similar HCDR1 insertions were also found in PGT122 HC variants (Figure S3C in Supplementary Material). Ultra-deep repertoire sequencing thus provided evidence that this HCDR1 insertion was likely a result of lineage diversification. Structural modeling of $\mathrm{Ab}_{\mathrm{d} 17}-2$ in complex with a native-like gp140 trimer (47) and an engineered gp120 outer domain revealed potential roles of this HCDR1 insertion (Figure 4F). As previously reported, the PGT121-class bNAbs utilize an open face formed by three HCDRs to interact with various components of the $\operatorname{Env}(37,41,42)$. Due to the close proximity of HCDR1 to HCDR2, some Env interactions might be shifted to HCDR1 as a means to accommodate the rapidly changing glycan shield. Indeed, the HCDR1 insertion site is $\sim 30 \AA$ from the glycan-rich V1, V3, and V4 loops, suggesting that an extended loop at this site may mediate interactions with multiple gp120 glycans.

In summary, digital panning of a diverse donor-17 scFv library identified bNAb-like scFv clones and a previously unknown HCDR1 variation within the PGT121 class. For comparative analysis, we also screened this scFv library against a clade-C V1V2 nanoparticle (48), which did not yield any neutralizing mAbs to the apex (Tables S2B and S3B, and Figures S4 and S5 in Supplementary Material), in line with the previous findings for this patient (34). Therefore, our results demonstrated a focused antibody repertoire tuned for specific recognition of a glycan supersite on the native Env.

\section{Digital Panning of a Focused Donor-17 ScFv Library Identifies bNAb Intermediates}

Previously, Sok et al. developed a novel phylogenetic method to derive putative intermediates of PGT121-134 based on the 454 sequencing data (18). These intermediates have been studied in detail to inform on the early events of bNAb development $(41,42)$ and to design trimer immunogens for sequential immunization of Ig knock-in mice $(23,31)$. Undoubtedly, patient samples will provide a more reliable source of bNAb precursors and intermediates, which, however, are only present at low frequencies within the memory B-cell repertoire. In this study, we hypothesized that digital panning of a bNAb-lineage-focused scFv library may capture these important but rare clones that would otherwise be inaccessible to the standard methods of antibody identification (96).

To test this hypothesis, we constructed a new scFv library from the donor-17 PBMCs using IgHV4- and IgLV3-specific primers to target the germline genes of the PGT121 class (Table S1 in 


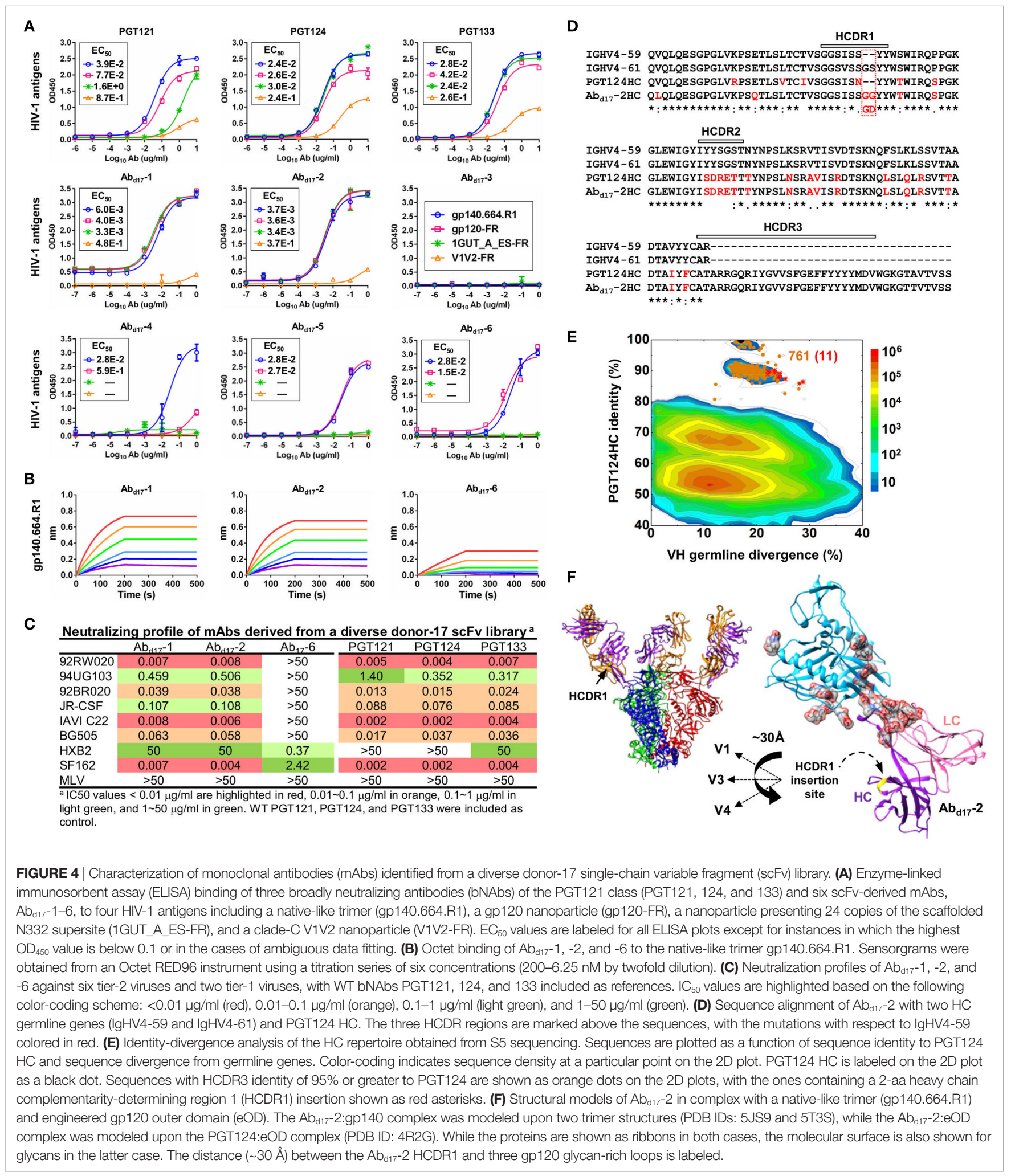

Supplementary Material). This library was screened against the trimer probe, with the pre- and post-panning libraries sequenced on the PGM using an Ion 314 v2 chip. NGS yielded 809,354 raw reads, which were analyzed with the H/L-paired Antibodyomics pipeline (Table S2C in Supplementary Material). Quantitative library profiles revealed convergence patterns similar to those observed for the diverse donor-17 scFv library (Figure 5A). In brief, IgHV4-59 and IgLV3-21 accounted for over $90 \%$ of 

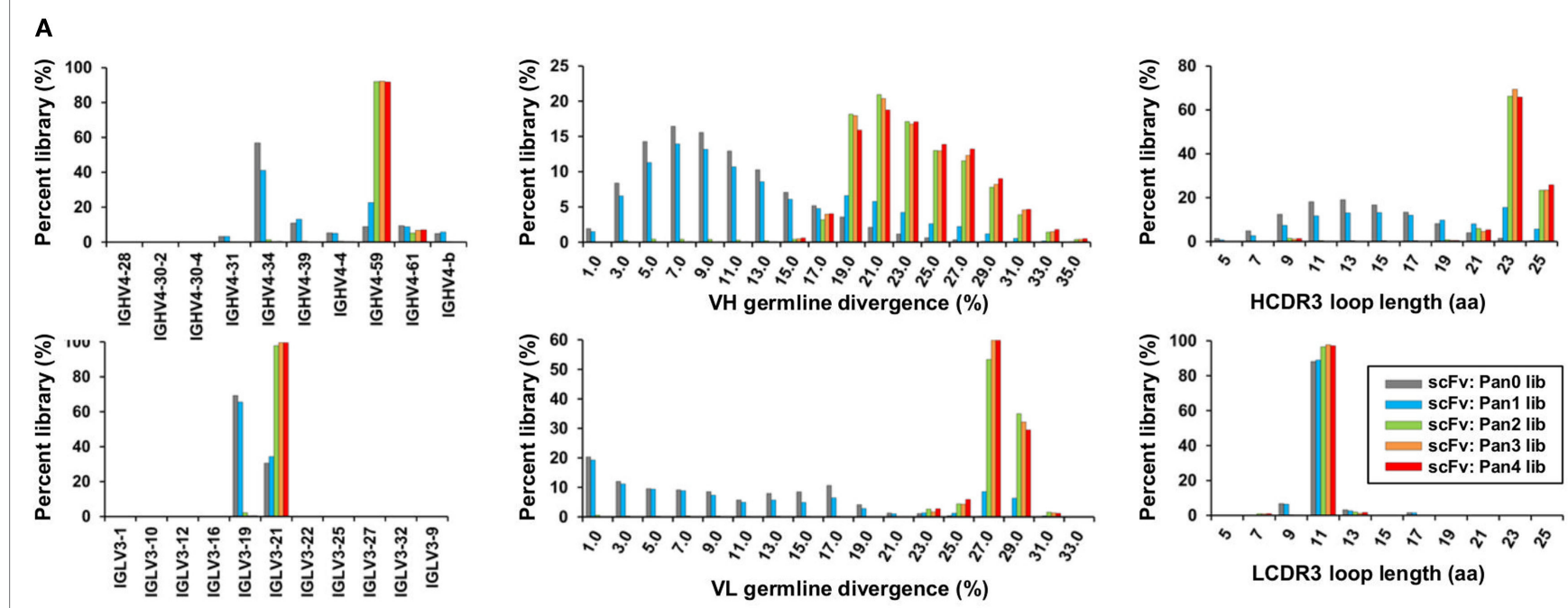

B
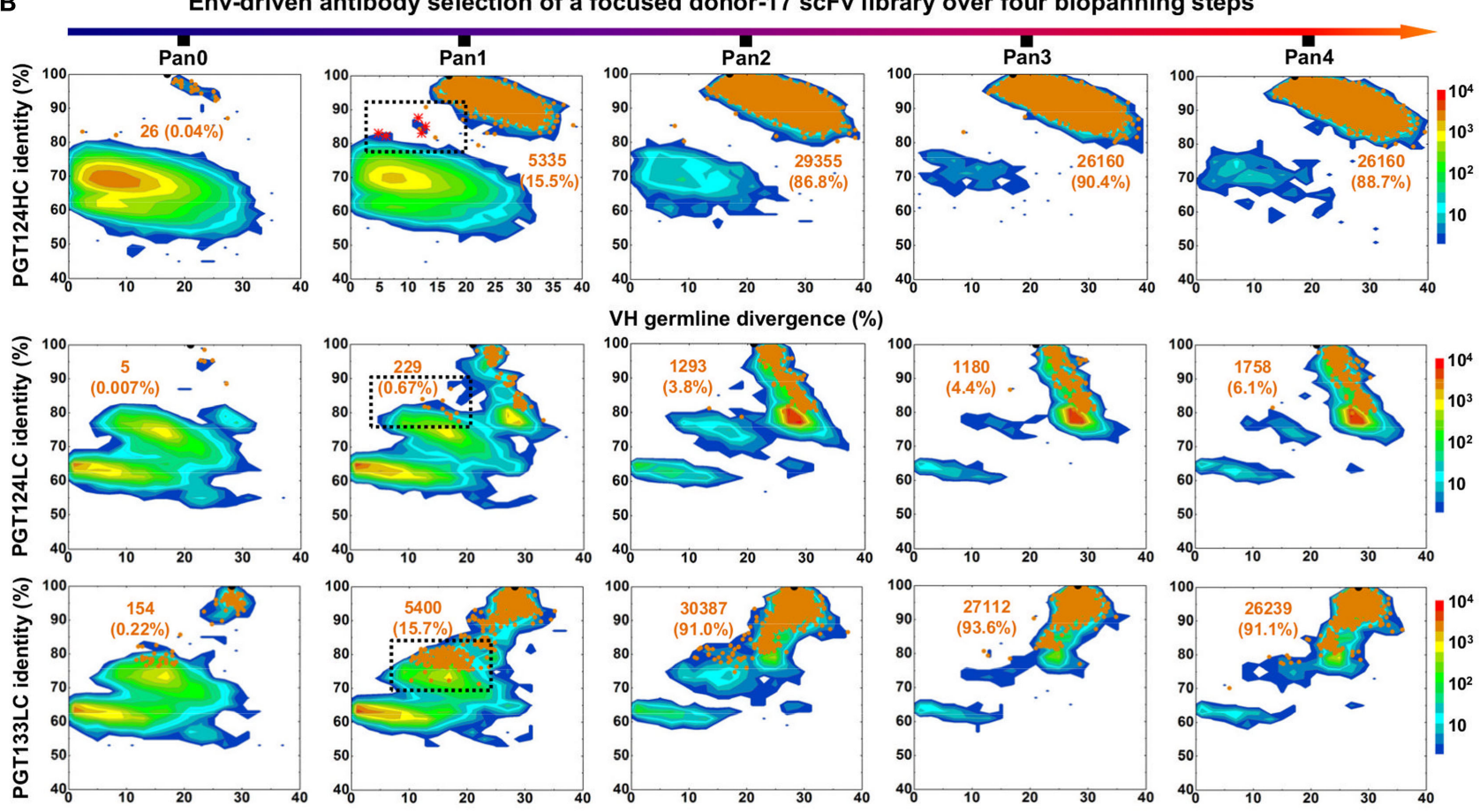

VH germline divergence $(\%)$
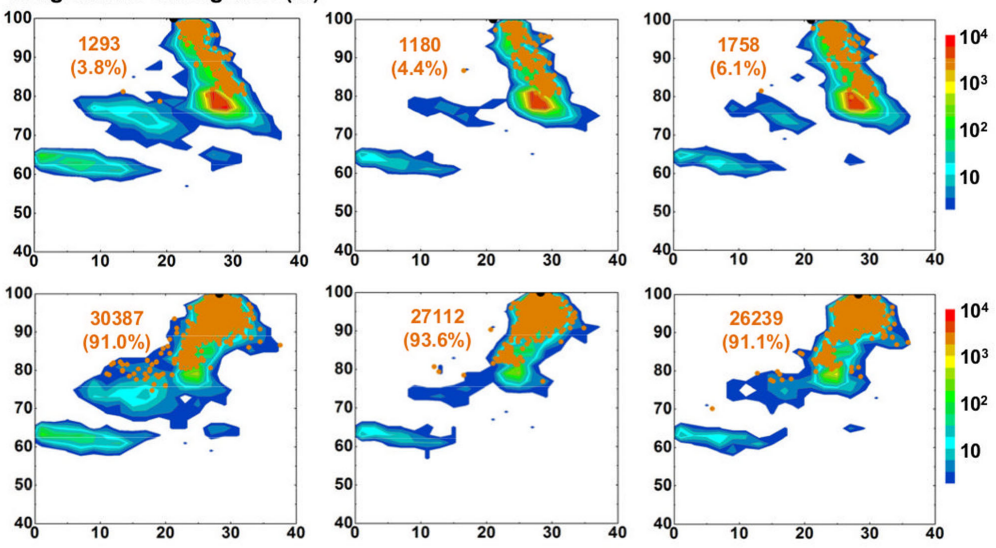

VL germline divergence $(\%)$

FIGURE 5 | Digital panning of a focused donor-17 single-chain variable fragment (scFv) library against the native-like trimer. A focused scFv library was constructed from donor-17 peripheral blood mononuclear cells (PBMCs) using lgHV4- and IgLV3-specific primers. (A) Distributions of germline gene usage (left), somatic hypermutation (middle), and CDR3 loop length (right) are plotted for the five scFv libraries obtained from the trimer panning process. Histograms are color-coded following the same scheme as in Figure 2B. (B) Identity-divergence analysis of the five donor-17 scFv libraries obtained from the trimer panning process. For each library, sequences are plotted as a function of sequence identity to PGT124 HC (top), PGT124 LC (middle), and PGT133 LC (bottom), and sequence divergence from germline genes. Color-coding indicates sequence density at a particular point on the 2D plot. WT PGT124 and PGT133 are labeled on the 2D plots as black dots. Sequences with CDR3 identity of 95\% or greater are shown as orange dots, with the number of sequences and library percentage labeled on the 2D plot for comparison. Sequences with low levels of SHM and moderate identity to the respective broadly neutralizing antibodies, corresponding to native intermediates (NINs), are circled by black dotted line boxes on the 2D plots for the Pan1 library. Five NIN-like scFv clones selected from the Pan1 library based on the HC identity to PGT124 are shown as red asterisks on the 2D plot.

HCs and LCs upon convergence. The average degree of SHM increased from $12 \%$ and $11 \%$ to $23 \%$ and $27 \%$ for HCs and LCs, respectively. In addition, more than $90 \%$ of HCs contained long
HCDR3 loops of 23-25 aa, characteristic of the PGT121-class bNAbs. Furthermore, the 2D plots demonstrated co-enrichment of PGT124-like HCs and PGT124/PGT133-like LCs, consistent 
with our findings for the diverse scFv library but with a notable difference in the pattern of distribution (Figure 5B). Nonetheless, the H/L-paired clustering analysis identified three representative $\mathrm{scFv}$ clones from the converged library that resembled the WT bNAbs (Figure 6A; Table S3C in Supplementary Material). While the scFv-derived $\mathrm{HC}$ database appeared to be enriched for PGT124-like sequences of the IgHV4-59 origin, the PGT133-like LCs were more prevalent than the PGT124-like LCs, showing an $\sim 16$-fold difference in their frequencies. Three mAbs $\left(\mathrm{Ab}_{\mathrm{d} 17}-7-9\right)$ were synthesized for evaluation of antigen binding by ELISA (Figure 6B). Similar to $A b_{d 17}-1$ and $A b_{d 17}-2$ identified from the diverse scFv library, $\mathrm{Ab}_{\mathrm{d} 17}-7$ and $A b_{\mathrm{d} 17}-8$ bound to the three N332-containing antigens with EC50 values 10- to 100 -fold lower than the WT bNAbs. Recognition of the native-like trimer by $\mathrm{Ab}_{\mathrm{d} 17}-7$ and $\mathrm{Ab}_{\mathrm{d} 17}-8$ was then confirmed by BLI (Figure 6C), which displayed binding profiles comparable to the WT bNAbs

A Representative scFv/mAb clones identified by H/L-paired, CDR3-based clustering analysis a

\begin{tabular}{|c|c|c|c|c|c|c|c|}
\hline Clone & $\mathrm{HC}$ name & VH identity & HCDR3 (aa) & Size & LC name & LCDR3 (aa) & Size \\
\hline $\mathrm{Ab}_{\mathrm{d} 17^{-7}}$ & T1P4H1 & & & & T1P4L1 & HYWDSRSPISWI & 23,502 \\
\hline$A b_{d 17}-8$ & T1P4H1 & $83.3 \%$ & ARRGQRIYGVVSFGEFFYYYYMDV & 25,597 & T1P4L2 & HMWDSRSGFSWS & 1,449 \\
\hline$A b_{\mathrm{d} 17}-9$ & $\mathrm{~T} 1 \mathrm{P} 4 \mathrm{H} 1$ & & & & T1P4L3 & QSYDSSLSGVV & 50 \\
\hline
\end{tabular}

a Listed items include scFv/mAb clone name, heavy chain (HC) name, VH germline gene identity (\%), HCDR3 sequence, HC

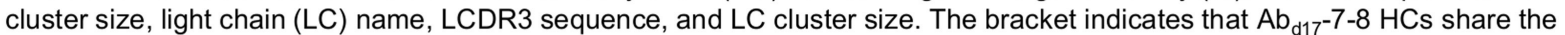
same HCDR3 sequences, thus belonging to a single HCDR3 cluster.
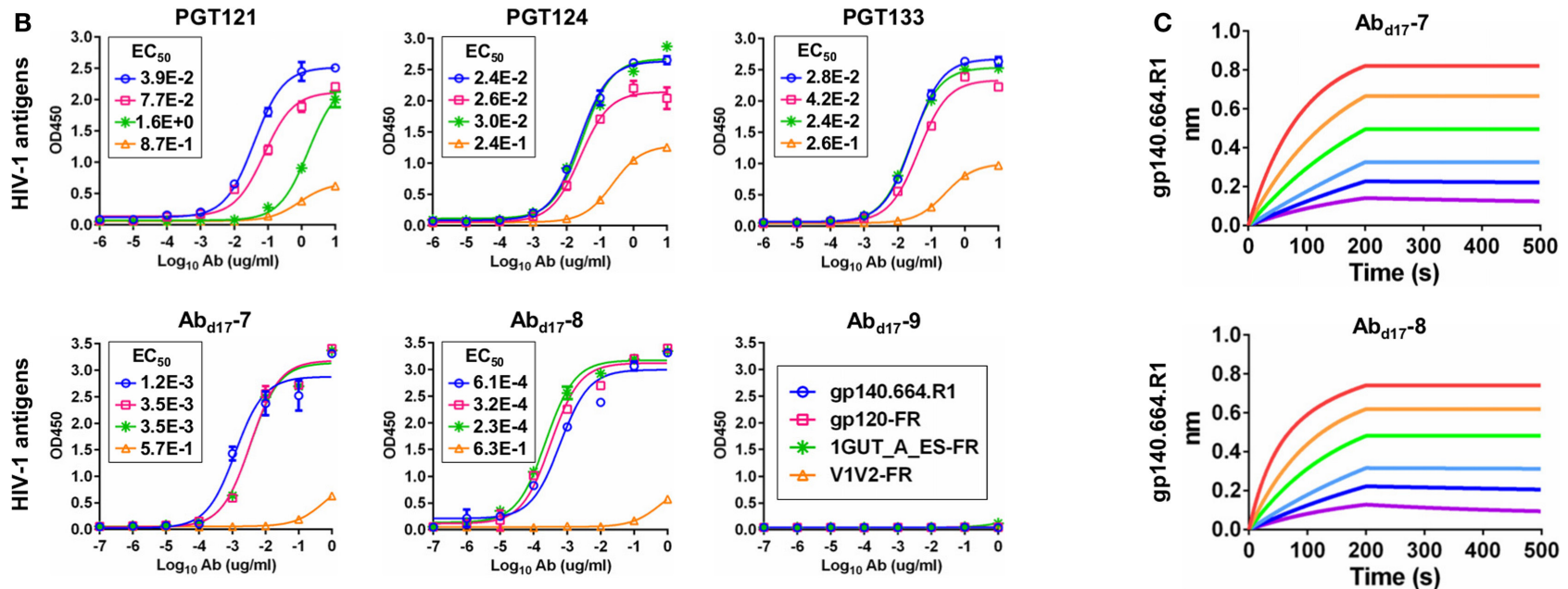

D Neutralizing profile of mAbs derived from a focused donor-17 scFv library a

\begin{tabular}{ccccccc}
\hline & $\mathrm{Ab}_{\mathrm{d} 17}-7$ & $\mathrm{Ab}_{\mathrm{d} 17}-8$ & & PGT121 & PGT124 & PGT133 \\
\cline { 1 - 2 } \cline { 5 - 7 } 92RW020 & 0.007 & 0.008 & & 0.005 & 0.004 & 0.007 \\
94UG103 & 0.358 & 0.318 & & 1.40 & 0.352 & 0.317 \\
92BR020 & 0.028 & 0.030 & & 0.013 & 0.015 & 0.024 \\
JR-CSF & 0.080 & 0.081 & & 0.088 & 0.076 & 0.085 \\
IAVI C22 & 0.006 & 0.005 & 0.002 & 0.002 & 0.004 \\
BG505 & 0.045 & 0.049 & & 0.017 & 0.037 & 0.036 \\
HXB2 & 50 & 50 & & $>50$ & $>50$ & 50 \\
SF162 & 0.003 & 0.005 & & 0.002 & 0.002 & 0.004 \\
MLV & $>50$ & $>50$ & $>50$ & $>50$ & $>50$ \\
\hline
\end{tabular}

a IC50 values $<0.01 \mu \mathrm{g} / \mathrm{ml}$ are highlighted in red, $0.01 \sim 0.1 \mu \mathrm{g} / \mathrm{ml}$ in orange, $0.1 \sim 1$ $\mu \mathrm{g} / \mathrm{ml}$ in light green, and 1 50 $\mu \mathrm{g} / \mathrm{ml}$ in green. WT PGT121, PGT124, and PGT133 were included for comparison.

FIGURE 6 | Characterization of monoclonal antibodies (mAbs) identified from a focused donor-17 single-chain variable fragment (scFv) library. (A) Representative scFv clones identified from a focused scFv library by H/L-paired, CDR3-based clustering analysis for mAb synthesis. (B) Enzyme-linked immunosorbent assay (ELISA) binding of three broadly neutralizing antibodies (bNAbs) of the PGT121 class (PGT121, 124, and 133) and three scFv-derived mAbs, Ab d17-7-9, to the gp140.664.R1 trimer and three nanoparticles (gp120-FR, 1GUT_A_ES-FR, and V1V2-FR). EC 50 values are labeled for all ELISA plots except for instances in which the highest $\mathrm{OD}_{450}$ value is below 0.1 or in the cases of ambiguous data fitting. (C) Octet binding of $\mathrm{Ab}_{\mathrm{d} 17}-7$ and -8 to the gp140.664.R1 trimer. Sensorgrams were obtained from an Octet RED96 instrument using a titration series of six concentrations (200-6.25 nM by twofold dilution). (D) Neutralization profiles of $\mathrm{Ab}_{\mathrm{d} 17}-7$ and -8 against six tier-2 viruses and two tier-1 viruses, with WT bNAbs PGT121, 124, and 133 included as references. $I_{50}$ values are highlighted based on the same color-coding scheme as in Figure $\mathbf{4 C}$. 
(Figure 1C). Consistently, $\mathrm{Ab}_{\mathrm{d} 17}-7$ and $\mathrm{Ab}_{\mathrm{d} 17}-8$ also demonstrated neutralizing breadth and potency almost identical to the PGT121class bNAbs with some weak activity against a tier-1 HXB2 strain, which could not be neutralized by the WT bNAbs (Figure 6D). Overall, screening of a focused donor-17 scFv library by a nativelike trimer probe resulted in mAbs with optimized binding properties and bNAb-like neutralization profiles.

Although trimer panning of two donor-17 scFv libraries converged to the bNAb-like clones with similar functions, visual comparison revealed "islands" of sequences present only on the $2 \mathrm{D}$ plots of the focused library, but not the diverse library (Figure 5B). To explore the cause of this discrepancy, we plotted the $\mathrm{HC}$ and LC sequences with a CDR3 identity of $95 \%$ or greater to the WT bNAbs. Surprisingly, these islands were mainly occupied by sequences with a low level of SHM $(<15 \%)$ and moderate identity to the WT bNAbs ( $~ 80 \%)$, suggesting that they may be the native intermediates (termed NINs) of the PGT121-class bNAbs. Interestingly, these NINs were most visible after the first panning step (termed Pan1) but began to disappear as the library was further enriched for high-affinity bNAb-like clones. Five NINs ( $\left.\mathrm{NIN}_{\mathrm{d} 17}-1-5\right)$ were selected from the Pan1 library for experimental testing (Figure 7A; Table S4 in Supplementary Material). Of note, sequence analysis revealed that the HCs of all five NINs contained the mature PGT124 HCDR3 loop with a low level of SHM (3-10\%) but were assigned to three different germline genes: IgHV4-34, IgHV4-59, and IgHV4-61. While $\mathrm{Ab}_{\mathrm{d} 17}-2$ and $\mathrm{NIN}_{\mathrm{d} 17}-2$ sharing the same IgHV4-61 germline gene with differing levels of SHM suggest an actively evolving PGT124 sub-lineage possessing the 2-aa insertion in HCDR1, the neargermline IgHV4-34 HCs with a mature PGT124 HCDR3 cannot be explained within the current framework of the PGT121-class bNAb lineage development. Due to the random HC/LC pairing in $\mathrm{scFv}$ library construction, diverse LCs were observed for the five NINs, with the PGT133-like LCs used by $\mathrm{NIN}_{\mathrm{d} 17}-4$ and -5 . Antigen binding of the five NIN-derived mAbs was then assessed by ELISA, with $\mathrm{NIN}_{\mathrm{d} 17-3}$ and -4 displaying notable affinities for the gp140.664.R1 trimer (47), the gp120 nanoparticle (48), and the N332 nanoparticle (Figure 7B) (46). To eliminate the effect of non-functional LCs, we paired the five NIN HCs with the PGT124 and PGT133 LCs. As expected, pairing with the WT bNAb LCs could restore the antigen affinity of $\mathrm{NIN}_{\mathrm{d} 17}-2$, -3 , and -4 , while moderately increasing the antigen affinity of $\mathrm{NIN}_{\mathrm{d} 17}-1$ and -5 . Lastly, a total of nine NIN-derived mAbs were tested for neutralization, with eight showing neutralizing activity (Figure 7C). Overall, NIN mAbs reconstituted from the IgHV4-59 and IgHV4-61 HCs outperformed those reconstituted from the IgHV4-34 HCs, exhibiting neutralization breadth on par with that of the WT bNAbs, although with reduced potency. Furthermore, the IgHV4-59 and IgHV4-61 HCs, when paired with the PGT124 LC, displayed more potent neutralization than their PGT133 LC counterparts. The results also suggested that an SHM level of $10 \%$ or lower may be sufficient for the PGT121class bNAb intermediates to achieve effective Env recognition (by targeting the N332 supersite) and broad HIV-1 neutralization.

Sequence alignment and structural modeling revealed distinct features of the newly derived PGT121-class intermediates (Figure 8). For example, compared with the WT PGT124 and an inferred PGT124 intermediate (32H) (18), NIN $_{\mathrm{d} 17-2-4}$ showed fewer mutations in the HCDR2 loop, suggesting that the highly mutated HCDR2 motif in the mature bNAbs may not be as critical as previously thought. Another important finding was an $N$-linked glycosylation site in the HCDR1 loop of $\mathrm{NIN}_{\mathrm{d} 17}-4$, suggesting that HCDR1 may be a focal point of maturation for facilitating Env interactions through various mechanisms. In contrast, the HCs of $\mathrm{NIN}_{\mathrm{d} 17}-1$ and -5 were IgHV4-34 germlinelike sequences with only four to six mutations within the $\mathrm{V}$ gene. Furthermore, while the HC mutations within the WT PGT124 and an inferred $32 \mathrm{H}$ (18) displayed similar distribution patterns across the protein surface, the $\mathrm{HC}$ mutations within $\mathrm{NIN}_{\mathrm{d} 17}-2$ and $\mathrm{NIN}_{\mathrm{d} 17}-4$ were focused mainly on HCDR1 of the functionally important open face (37). Additionally, we identified the NIN-like PGT124 and PGT133 LCs, which contained a similar deletion in FR1 but lacked the 3-aa FR3 insertion compared to the mature bNAbs (Figure S6 in Supplementary Material).

Our results confirmed that PGT124, and a related 10-1074 (36), represents a distinct branch of lineage development with respect to other members of the PGT121 class (18, 40-42), and define the minimal SHM needed to achieve broad HIV-1 neutralization. Since PGT124 and 10-1074 mainly require the N332 glycan for Env recognition $(42,45)$, and PGT124 intermediates with different levels of SHM have been found in the donor repertoire, the PGT124 sub-lineage may provide a more promising template than PGT121 for immunogen design targeting the N332 supersite.

\section{PGT124 Intermediates Compete with Trimer-Elicited Mouse Sera for the N332 Supersite}

Since the unmutated SOSIP.664 trimer was poorly recognized by the PGT121 precursor, random mutagenesis and structure-based design were undertaken to create sequential trimer immunogens to target the inferred PGT121 precursor and intermediates $(18,31)$. Although these modified trimers induced bNAb-like responses in Ig knock-in mice (23), they have not been tested in WT animals. In this study, PGT124 intermediates were selected by a native-like trimer probe presenting an intact glycan shield. Based on this finding, we hypothesized that an unmutated trimer may be able to elicit N332-speficic antibody responses in WT animals and that the bNAb intermediates (NINs) identified from the donor-17 scFv library can in turn be used in a competition assay to assess the N332 specificity of serum antibodies elicited by a trimer, or an N332-focused immunogen.

We briefly investigated this hypothesis by examining serum samples from a previous mouse immunization aimed to study the early B-cell responses to the N332 supersite and MPER in the context of multivalent scaffolds and native-like trimers (46). It was reported that a scaffolded full-length gp140 trimer elicited a robust antibody response to the apex, suggesting that the inclusion of MPER and a C-terminal scaffold domain can stabilize the glycan shield and facilitate bNAb recognition (46). In this study, we first performed ELISA to assess sera from mice immunized with the gp140.664.R1 trimer, which was also the basis of the trimer probe used for screening the donor-17 scFv libraries (Figure 9A). Consistent with the previous finding for the SOSIP trimer (102) 


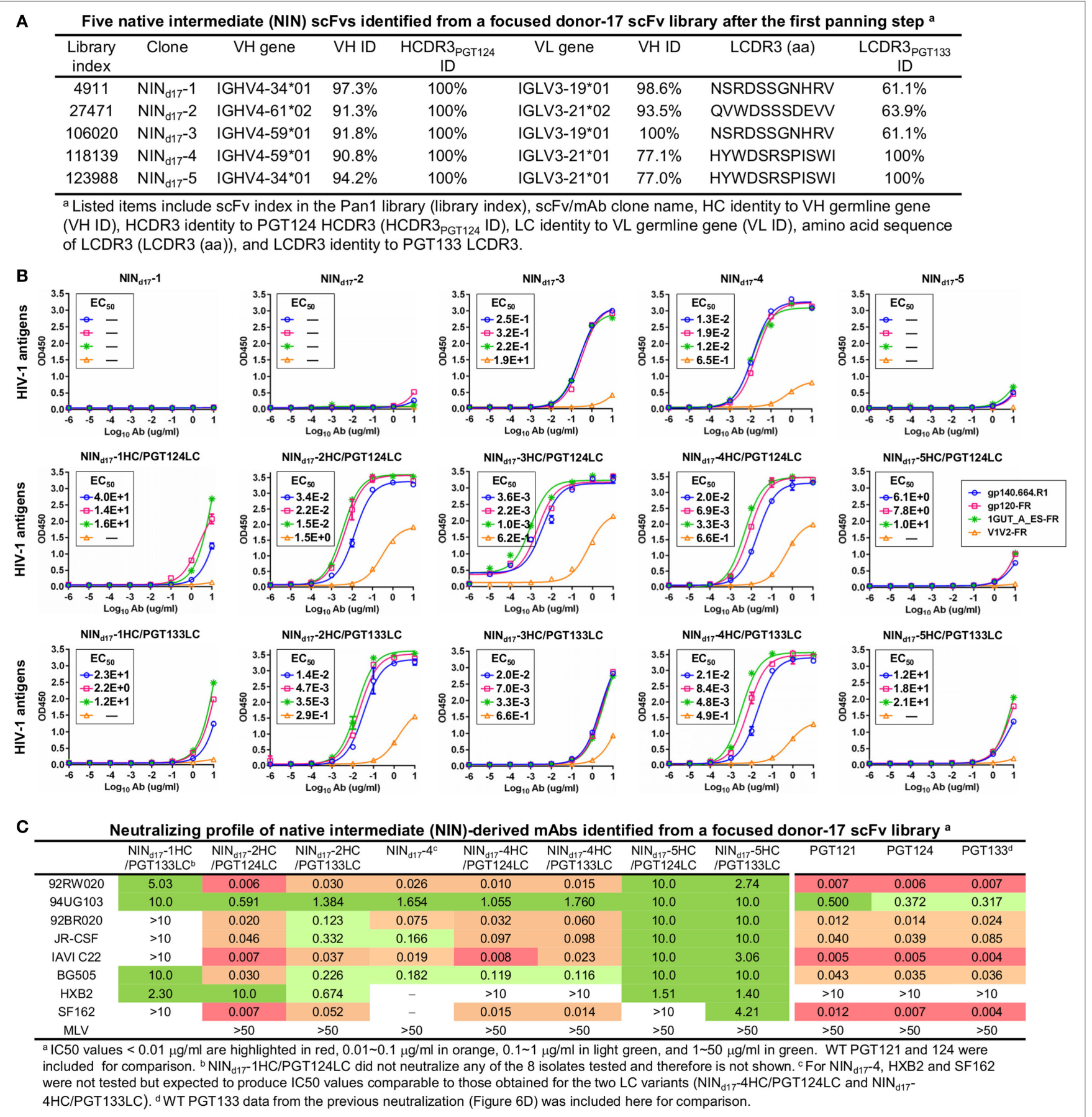

FIGURE 7 | Characterization of the PGT124 intermediates identified from a focused donor-17 single-chain variable fragment (scFv) library. (A) Five native intermediates, $\mathrm{NIN}_{\mathrm{d} 17}-1-5$, identified from the Pan1 library based on the HCDR3 analysis. (B) Enzyme-linked immunosorbent assay (ELISA) binding of NIN $\mathrm{d}_{17}-1-5$ and their LC variants to the gp140.664.R1 trimer and three nanoparticles (gp120-FR, 1GUT_A_ES-FR, and V1V2-FR). The two sets of LC variants were derived by pairing the NIN HCs with PGT124 and PGT133 LCs. EC 50 values are labeled for all ELISA plots except for instances in which the highest OD ${ }_{450}$ value is below 0.1 or in the cases of ambiguous data fitting. (C) Neutralization profiles of selected NIN $\mathrm{d}_{\mathrm{d} 17}$ variants against six tier-2 viruses and two tier-1 viruses, with WT broadly neutralizing antibodies PGT121, 124, and 133 included as references. IC Fo $_{50}$ values are highlighted based on the same color-coding scheme as in Figure $\mathbf{4 C}$.

and with our previous experiment (46), the apex and the N332 supersite were not well recognized by mouse sera, as indicated by two nanoparticle probes. In comparison, enhanced binding to both glycan epitopes was observed for mouse sera elicited by the scaffolded gp140.681.R1-1NOG trimer (Figure 9B), suggesting that an unmutated trimer, when presented in a proper structural context, can induce antibody responses to the bNAb epitopes in the glycan shield. Two library-derived bNAb intermediates, 


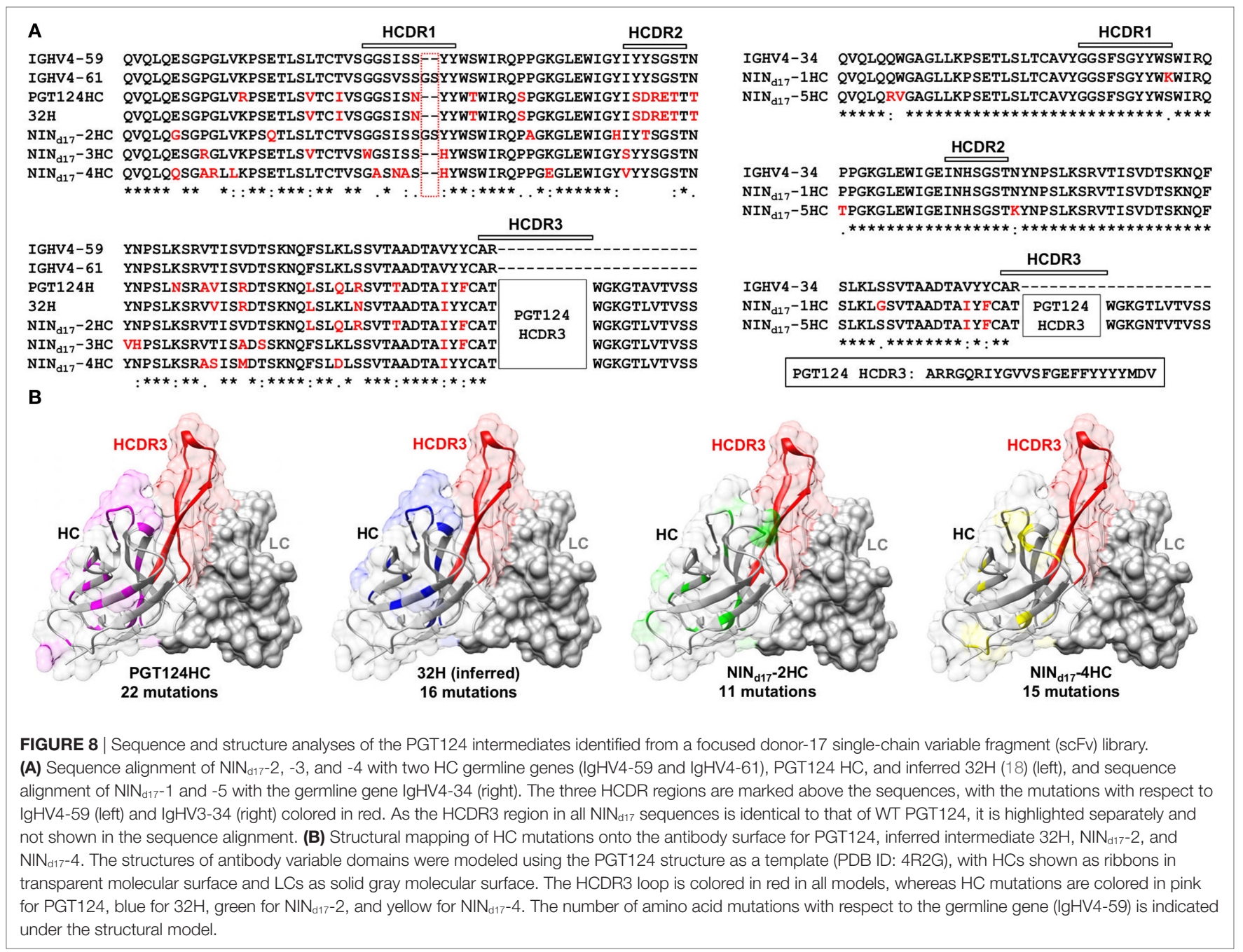

$\mathrm{NIN}_{\mathrm{d} 17}-1 \mathrm{HC} / \mathrm{PGT133LC}$ (with weak trimer binding and neutralization) and $\mathrm{NIN}_{\mathrm{d} 17}-4$ (with moderate neutralizing potency), were utilized in a competition ELISA to assess the N332 specificity of mouse sera obtained from the scaffolded trimer group (Figure 9C). The WT PGT124 was included in the ELISA assay as a positive control. As expected, PGT124 and $\mathrm{NIN}_{\mathrm{d} 17}-4$ - but not the $\mathrm{NIN}_{\mathrm{d} 17}-1$ variant with a near-germline $\mathrm{HC}$ - could effectively block serum binding to the N332 nanoparticle, illustrating the utility of the library-derived bNAb intermediates for immunogen evaluation. It also appeared that murine antibodies, with short (on average 9-aa) HCDR3 loops, failed to compete with human bNAb intermediates with a 24 -aa HCDR3 loop poised to penetrate the glycan shield.

\section{DISCUSSION}

Diverse bNAbs identified from the elite neutralizers have served as useful templates for guiding rational HIV-1 vaccine design (103). However, there is a significant disparity between the degrees of SHM observed for bNAbs and for weak- or non-NAb antibody responses in chronic infection and vaccination. The minimal level of SHM required for neutralization and protection is poorly defined, although recent studies of bNAb-virus co-evolution have begun to shed some light on this critical issue $(16,17,19-22)$. Limited by patient samples and antibody technologies, it remains a challenge to identify bNAb precursors and intermediates from infected donors, thus hindering B-cell lineage immunogen design (7). To overcome this challenge, germline reversion and phylogenic analysis have been used to derive putative bNAb precursors and intermediates (18). Analyses of the inferred antibodies have revealed distinct patterns for the PGT121 and PGT124 sub-lineages (40-42). These insights constituted the foundation for designing sequential trimer constructs capable of eliciting bNAb-like responses in Ig knock-in mice $(23,31)$. Despite these encouraging successes, it is necessary to acknowledge the risk in utilizing inferred antibodies resulting from assumptions which may or may not accurately reflect the true events of B-cell lineage development.

A variety of methods has been applied to the identification of HIV-1 bNAbs, including phage display, hybridoma, single B-cell culturing coupled with large-scale functional screening, and antigen-specific single B-cell sorting by flow cytometry 


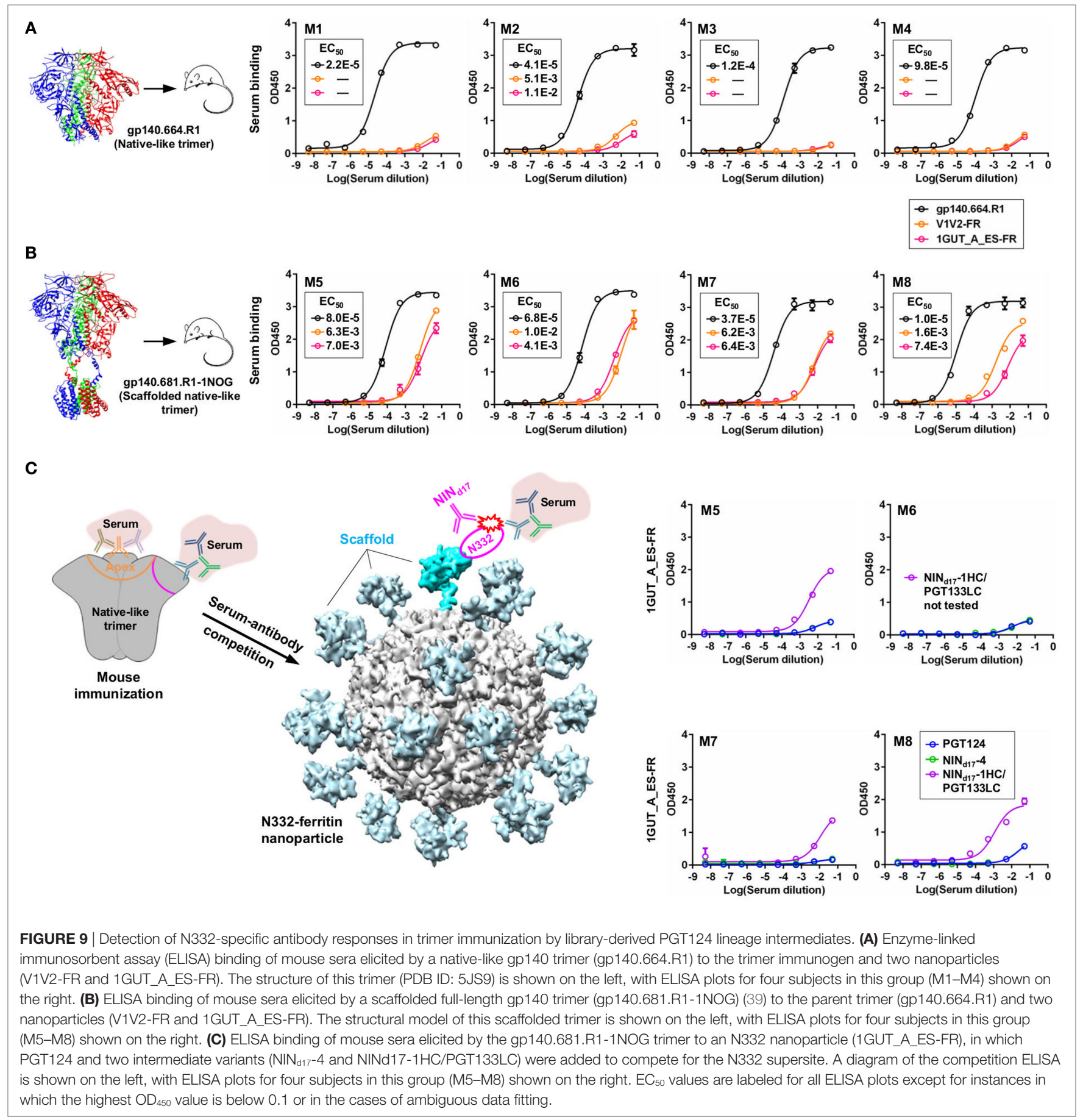

(96). Among these, the single-cell methods are considered most advantageous, as they enable isolation of mAbs with natively paired HC and LC from live, functional B cells. It was also suggested that new methods would be necessary for identifying rare precursors and intermediates - a challenge facing both bNAb and immunization studies (96). Advances in NGS technology and templating methods have allowed unbiased analysis of B-cell repertoires during HIV-1 infection and vaccination $(15,17,46,101)$. Presently, NGS is applied to the characterization of scFv antibody libraries but not yet to the direct selection of functional $\mathrm{scFv}$ clones due to its insufficient lead length to cover both HC and LC within a scFv (100). In this study, a long-read NGS technology was established that permitted high-throughput sequencing of full-length scFv libraries and used in conjunction with an H/L-paired antibodyomics method for library profiling and clone selection. These advances have transformed the conventional scFv library panning method into a quantitative "digital panning" method and will likely improve the single-cell, 
H/L-paired antibody isolation and repertoire analysis $(104,105)$. Using two scFv libraries constructed from the samples of an elite HIV-1 neutralizer (donor-17) (34), we demonstrated the utility of digital panning in the study of PGT121-class bNAbs, with due consideration of recent advances in gp140 trimer design $(86,87)$. A native-like trimer probe, with structural and antigenic profiles indistinguishable from its parent UFO trimer (47), was utilized to screen donor-17 scFv libraries so as to dissect the details of an N332-dependent bNAb lineage within the antibody repertoire. Digital panning of donor-17 scFv libraries with this trimer probe offered a wealth of novel information on the PGT121 class: PGT122/PGT124 HC variants with a 2-aa HCDR1 insertion, $\mathrm{HC}$ and LC intermediates with fewer mutations and different sequence motifs than the inferred intermediates (18), and a versatile open face utilizing HCDR1 and HCDR2 mutations to mediate Env interactions. However, due to the shortcomings of phage display such as primer bias and random $\mathrm{H} / \mathrm{L}$ pairing, it is possible that only a subset of lineage variants and intermediates were captured. Nonetheless, these new findings will contribute to a more complete understanding of the lineage development of the PGT121-class bNAbs. The bNAb intermediates were also utilized to gauge N332-specific antibody responses elicited by two native-like gp140 trimers. A previously reported gp140 scaffolding strategy (46) appeared to notably enhance the trimerinduced antibody response to the $\mathrm{N} 332$ supersite in BALB/c mice, although such response could barely compete with the human $\mathrm{bNAb}$ intermediates. Future studies investigating the cause of this enhancement, or evaluating the scaffolded trimers using an extensive regimen, may prove useful for the development of an effective trimer vaccine.

In summary, our study has provided valuable tools, including a native-like trimer probe and the digital panning method, to facilitate bNAb studies, particularly those involving identification of rare bNAb intermediates in HIV-1 patient and vaccination samples. The PGT124 sub-lineage, possessing an invariable HCDR3 loop and multiple library-derived intermediates, may serve as a promising template for $\mathrm{B}$-cell lineage vaccine design targeting the N332 supersite.

\section{ETHICS STATEMENT}

Blood samples were acquired from an HIV-1-infected donor (donor-17) of the Protocol G cohort under written consent. The samples were collected following clinical protocols approved by the Republic of Rwanda National Ethics Committee, the Emory University Institutional Review Board, the University of Zambia Research Ethics Committee, the Charing Cross Research Ethics Committee, the UVRI Science and Ethics Committee, the University of New South Wales Research Ethics Committee, the St. Vincent's Hospital and Eastern Sydney Area Health Service, the Kenyatta National Hospital Ethics and Research Committee, the University of Cape Town Research Ethics Committee, the International Institutional Review Board, the Mahidol University Ethics Committee, the Walter Reed Army Institute of Research (WRAIR) Institutional Review Board, and the Ivory Coast Comité National d'Ethique des Sciences de la Vie et de la Santé (CNESVS).

\section{AUTHOR CONTRIBUTIONS}

LH and JZ conceived the method. LH, XL, PA, CJM, RA, CDM, and JZ performed digital panning, antibody synthesis, antigen binding assays, and computational analysis. NV, GO, and AW performed negative-stain EM analysis of the gp140 trimer probe. BZ set up the MicroPulser system. KS-F, DS, and DB performed the HIV-1 neutralization assays. LH and JZ wrote the manuscript with input from all coauthors.

\section{ACKNOWLEDGMENTS}

Electron microscopy data were collected at the Scripps Research Institute EM Facility.

\section{FUNDING}

This work was partly supported by the International AIDS Vaccine Initiative Neutralizing Antibody Center and CAVD, by the Center for HIV/AIDS Vaccine Immunology and Immunogen Discovery (CHAVI-ID UM1 AI00663) (AW, DB), by the HIV Vaccine Research and Design (HIVRAD) program (P01 AI110657) (AW), and by the HIV Vaccine Research and Design (HIVRAD) program [P01 AI124337 (JZ), AI084817 (AW), AI129698 (JZ)], and AI125078-01A1 (JZ).

\section{SUPPLEMENTARY MATERIAL}

The Supplementary Material for this article can be found online at http://journal.frontiersin.org/article/10.3389/fimmu.2017.01025/ full\#supplementary-material.

FIGURE S1 | Negative-stain EM of the biotinylated, Avi-tagged BG505 gp140 trimer probe used for biopanning. This trimer probe contains a redesigned heptad repeat 1 bend (47) and a biotinylated Avi-tag located immediately downstream of residue 664 (termed gp140.664.R1-Avi-Biot). (A) Raw micrograph of the BG505 gp140.664.R1-Avi-Biot trimer. (B) Reference-free 2D class averages of the BG505 gp140.664.R1-Avi-Biot trimer. Percentages of native-like trimers (closed and partially open or breathing trimers) and non-native species (misfolded trimers as well as dimers and monomers) are indicated. (C) The estimated resolution $(\sim 21 \AA)$ of the EM reconstruction was calculated from the Fourier Shell Correlation (FSC) using a cutoff of 0.5. (D) Top and side views of the 3D EM reconstruction of the BG505 gp140 SOSIP.664 trimer and the gp140.664.R1-Avi-Biot trimer. The EM densities of the SOSIP trimer are shown in gray transparent surface with the crystal structure (PDB 4TVP, gp120 in blue with V1V2 in magenta, V3 in green and gp41 in brown) fitted into the density. The EM densities of the gp140.664. R1-Avi-Biot trimer are shown in gray transparent surface with the SOSIP trimer densities overlaid as wire mesh (in orange). The contour level used for the gp140.664.R1-Avi-Biot trimer density was $\sim 33$.

FIGURE S2 | Assessment of the Antibodyomics 2.0 pipeline. (A) Schematic view of the chain-specific Antibodyomics pipeline, which consists of five steps including (1) data reformatting and cleaning, (2) germline gene assignment, (3) sequencing error correction, (4) calculation of sequence identity to a set of known antibodies, and (5) determination of CDR3 and variable domain boundaries. Step 3 (error correction) is highlighted with a red dashed-line box. (B) Distribution of improvement in sequence quality resulting from error correction. Quality improvement is measured by the change of amino acid sequence identity with respect to the germline $\mathrm{V}$ gene. For the donor-17 antibody chain data generated by 454 sequencing (18), Antibodyomics 1.0 yielded an average improvement of 19.4 and $21.7 \%$ for $\mathrm{HC}$ and LC, respectively, in comparison with 22.5 and $24.1 \%$ from Antibodyomics 2.0 . 
FIGURE S3 | Ultra-deep sequencing of the donor-17 $\mathrm{HC}$ repertoire.

(A) Distributions of germline gene usage (left), somatic hypermutation (middle), and HCDR3 length (right). In the histogram of germline gene family distribution, IgHV4 is highlighted in black whereas other germline genes are shown in gray (left 1). A more detailed distribution within the IgHV4 family is also plotted (left 2). (B) Identity-divergence analysis of the donor-17 HC repertoire using the PGT121class bNAb HCs as templates. Sequences are plotted as a function of sequence identity to WT bNAb HCs and germline divergence. Color-coding indicates sequence density at a particular point on the 2D plot. Wild-type bNAb HCs are labeled on the 2D plots as black dots. Sequences with HCDR3 identity of $90 \%$ or greater and those assigned to IgHV4-61 are shown as orange dots and red asterisks, respectively, with the number of sequences for each labeled on the 2D plot. (C) Sequence alignment of selected HCs of the putative IgHV4-61 origin with respect to two germline genes (IgHV4-59 and IgHV4-61) and WT bNAb $\mathrm{HCs}$. The three HCDR regions are marked above the sequences, with the mutations with respect to IgHV4-59 colored in red. Below the sequence alignment, asterisk $\left(^{*}\right)$ indicates identical residues, colon (:) indicates residues with strongly similar properties, and period (.) indicates residues with weakly similar properties.

FIGURE S4 | Digital panning of a diverse donor-17 single-chain variable fragment (scFv) library against a clade-C V1V2-ferritin nanoparticle. This scFv library, constructed from the donor-17 peripheral blood mononuclear cells (PBMCs) using a large set of primers, has been screened against a native-like gp140 trimer probe, gp140.664.R1-Avi-Biot. Distributions of germline gene usage (A), somatic hypermutation (B), and CDR3 loop length (C) are plotted for

\section{REFERENCES}

1. Kwong PD, Mascola JR. Human antibodies that neutralize HIV-1: identification, structures, and B cell ontogenies. Immunity (2012) 37:412-25. doi:10.1016/j.immuni.2012.08.012

2. Kwong PD, Mascola JR, Nabel GJ. Broadly neutralizing antibodies and the search for an HIV-1 vaccine: the end of the beginning. Nat Rev Immunol (2013) 13:693-701. doi:10.1038/nri3516

3. Burton DR, Mascola JR. Antibody responses to envelope glycoproteins in HIV-1 infection. Nat Immunol (2015) 16:571-6. doi:10.1038/ni.3158

4. Klein F, Mouquet H, Dosenovic P, Scheid JF, Scharf L, Nussenzweig MC. Antibodies in HIV-1 vaccine development and therapy. Science (2013) 341:1199-204. doi:10.1126/science. 1241144

5. Walker LM, Burton DR. Rational antibody-based HIV-1 vaccine design: current approaches and future directions. Curr Opin Immunol (2010) 22:358-66. doi:10.1016/j.coi.2010.02.012

6. Burton DR, Ahmed R, Barouch DH, Butera ST, Crotty S, Godzik A, et al. A blueprint for HIV vaccine discovery. Cell Host Microbe (2012) 12:396-407. doi:10.1016/j.chom.2012.09.008

7. Haynes BF, Kelsoe G, Harrison SC, Kepler TB. B-cell-lineage immunogen design in vaccine development with HIV-1 as a case study. Nat Biotechnol (2012) 30:423-33. doi:10.1038/nbt.2197

8. Wu X, Zhou T, Zhu J, Zhang B, Georgiev I, Wang C, et al. Focused evolution of HIV-1 neutralizing antibodies revealed by structures and deep sequencing. Science (2011) 333:1593-602. doi:10.1126/science.1207532

9. Zhu J, O'Dell S, Ofek G, Pancera M, Wu X, Zhang B, et al. Somatic populations of PGT135-137 HIV-1-neutralizing antibodies identified by 454 pyrosequencing and bioinfornnatics. Front Microbiol (2012) 3:315. doi:10.3389/ fmicb.2012.00315

10. Zhou T, Zhu J, Wu X, Moquin S, Zhang B, Acharya P, et al. Multidonor analysis reveals structural elements, genetic determinants, and maturation pathway for HIV-1 neutralization by VRC01-class antibodies. Immunity (2013) 39:245-58. doi:10.1016/j.immuni.2013.04.012

11. Zhu J, Ofek G, Yang Y, Zhang B, Louder MK, Lu G, et al. Mining the antibodyome for HIV-1-neutralizing antibodies with next-generation sequencing and phylogenetic pairing of heavy/light chains. Proc Natl Acad Sci USA (2013) 110:6470-5. doi:10.1073/pnas.1219320110

12. Zhu J, Wu X, Zhang B, McKee K, O’Dell S, Soto C, et al. De novo identification of VRC01 class HIV-1-neutralizing antibodies by next-generation the five scFv libraries obtained from the nanoparticle panning process. Histograms are color-coded according to their antigen panning steps: gray (Pan0), cyan (Pan1), green (Pan2), orange (Pan3), and red (Pan4).

FIGURE S5 | Identification and characterization of monoclonal antibodies (mAbs) from a diverse donor-17 single-chain variable fragment (scFv) library screened against a clade-C V1V2-ferritin nanoparticle. (A) Six prevalent scFv clones identified by $\mathrm{H} / \mathrm{L}$-paired, CDR3-based clustering analysis. (B) Enzyme-linked immunosorbent assay (ELISA) binding of three representative bNAbs of the PGT121 class (PGT121, 124, and 133) and six scFv-derived mAbs (VAb d17 $_{1-1-6)}$ to four HIV-1 antigens including a native-like trimer (gp140.664.R1), a gp120ferritin nanoparticle (gp120-FR), an N332 nanoparticle (1GUT_A_ES-FR), and a V1V2-ferritin nanoparticle (V1V2-FR). For $V A b_{d 17}$, ferritin was included in the ELISA as a negative control. EC Fo $_{50}$ values are labeled for all ELISA plots except for instances in which the highest $\mathrm{OD}_{450}$ value is below 0.1 or in the cases of ambiguous data fitting.

FIGURE S6 | Additional native intermediates (NINs) selected from a focused donor-17 single-chain variable fragment (scFv) library during the trimer panning process. (A) Sequence alignment of HCs with assigned germline genes and WT PGT124 HC. (B) Sequence alignment of LCs with germline gene IgLV3-21 and WT PGT124 LC or PGT133 LC. The three HCDR regions are marked above the sequences, with the mutations with respect to the assigned germline gene colored in red. Below the sequence alignment, asterisk $\left(^{*}\right)$ indicates identical residues, colon (:) indicates residues with strongly similar properties, and period (.) indicates residues with weakly similar properties.

sequencing of B-cell transcripts. Proc Natl Acad Sci USA (2013) 110:E4088-97. doi:10.1073/pnas.1306262110

13. Wu X, Zhang Z, Schramm CA, Joyce MG, Kwon YD, Zhou T, et al. Maturation and diversity of the VRC01-antibody lineage over 15 years of chronic HIV-1 infection. Cell (2015) 161:470-85. doi:10.1016/j.cell.2015.03.004

14. Sok D, Pauthner M, Briney B, Lee JH, Saye-Francisco KL, Hsueh J, et al. A prominent site of antibody vulnerability on HIV envelope incorporates a motif associated with CCR5 binding and its camouflaging glycans. Immunity (2016) 45:31-45. doi:10.1016/j.immuni.2016.06.026

15. He L, Sok D, Azadnia P, Hsueh J, Landais E, Simek M, et al. Toward a more accurate view of human B-cell repertoire by next-generation sequencing, unbiased repertoire capture and single-molecule barcoding. Sci Rep (2014) 4:6778-6778. doi:10.1038/srep06778

16. Doria-Rose NA, Schramm CA, Gorman J, Moore PL, Bhiman JN, DeKosky BJ, et al. Developmental pathway for potent V1V2-directed HIV-neutralizing antibodies. Nature (2014) 509:55-62. doi:10.1038/nature13036

17. Kong L, Ju B, Chen Y, He L, Ren L, Liu J, et al. Key gp120 glycans pose roadblocks to the rapid development of VRC01-class antibodies in an HIV-1-infected Chinese donor. Immunity (2016) 44:939-50. doi:10.1016/j.immuni.2016.03.006

18. Sok D, Laserson U, Laserson J, Liu Y, Vigneault F, Julien JP, et al. The effects of somatic hypermutation on neutralization and binding in the PGT121 family of broadly neutralizing HIV antibodies. PLoS Pathog (2013) 9:e1003754. doi:10.1371/journal.ppat.1003754

19. MacLeod DT, Choi NM, Briney B, Garces F, Ver LS, Landais E, et al. Early antibody lineage diversification and independent limb maturation lead to broad HIV-1 neutralization targeting the Env high-mannose patch. Immunity (2016) 44:1215-26. doi:10.1016/j.immuni.2016.04.016

20. Gao F, Bonsignori M, Liao HX, Kumar A, Xia SM, Lu X, et al. Cooperation of B cell lineages in induction of HIV-1-broadly neutralizing antibodies. Cell (2014) 158:481-91. doi:10.1016/j.cell.2014.06.022

21. Liao HX, Lynch R, Zhou T, Gao F, Alam SM, Boyd SD, et al. Co-evolution of a broadly neutralizing HIV-1 antibody and founder virus. Nature (2013) 496:469-76. doi:10.1038/nature 12053

22. Bonsignori M, Zhou T, Sheng Z, Chen L, Gao F, Joyce MG, et al. Maturation pathway from germline to broad HIV-1 neutralizer of a CD4-mimic antibody. Cell (2016) 165:449-63. doi:10.1016/j.cell.2016.02.022

23. Escolano A, Steichen JM, Dosenovic P, Kulp DW, Golijanin J, Sok D, et al. Sequential immunization elicits broadly neutralizing anti-HIV-1 antibodies in Ig knockin mice. Cell (2016) 166:1445-58. doi:10.1016/j.cell.2016.07.030 
24. Briney B, Sok D, Jardine JG, Kulp DW, Skog P, Menis S, et al. Tailored immunogens direct affinity maturation toward HIV neutralizing antibodies. Cell (2016) 166:1459-70. doi:10.1016/j.cell.2016.08.005

25. Jardine J, Julien JP, Menis S, Ota T, Kalyuzhniy O, McGuire A, et al. Rational HIV immunogen design to target specific germline B cell receptors. Science (2013) 340:711-6. doi:10.1126/science.1234150

26. Jardine JG, Sok D, Julien JP, Briney B, Sarkar A, Liang CH, et al. Minimally mutated HIV-1 broadly neutralizing antibodies to guide reductionist vaccine design. PLoS Pathog (2016) 12(8):e1005815. doi:10.1371/journal. ppat. 1005815

27. McGuire AT, Gray MD, Dosenovic P, Gitlin AD, Freund NT, Petersen J, et al. Specifically modified Env immunogens activate B-cell precursors of broadly neutralizing HIV-1 antibodies in transgenic mice. Nat Commun (2016) 7:10618. doi:10.1038/ncomms10618

28. McGuire AT, Hoot S, Dreyer AM, Lippy A, Stuart A, Cohen KW, et al. Engineering HIV envelope protein to activate germline B cell receptors of broadly neutralizing anti-CD4 binding site antibodies. J Exp Med (2013) 210:655-63. doi:10.1084/jem.20122824

29. Tian M, Cheng C, Chen X, Duan H, Cheng HL, Dao M, et al. Induction of HIV neutralizing antibody lineages in mice with diverse precursor repertoires. Cell (2016) 166:1471-84. doi:10.1016/j.cell.2016.07.029

30. Jardine JG, Ota T, Sok D, Pauthner M, Kulp DW, Kalyuzhniy O, et al. Priming a broadly neutralizing antibody response to HIV-1 using a germline-targeting immunogen. Science (2015) 349:156-61. doi:10.1126/science.aac5894

31. Steichen JM, Kulp DW, Tokatlian T, Escolano A, Dosenovic P, Stanfield RL, et al. HIV vaccine design to target germline precursors of glycan-dependent broadly neutralizing antibodies. Immunity (2016) 45:483-96. doi:10.1016/j. immuni.2016.08.016

32. Doores KJ. The HIV glycan shield as a target for broadly neutralizing antibodies. FEBS J (2015) 282:4679-91. doi:10.1111/febs.13530

33. Crispin M, Doores KJ. Targeting host-derived glycans on enveloped viruses for antibody-based vaccine design. Curr Opin Virol (2015) 11:63-9. doi:10.1016/j.coviro.2015.02.002

34. Walker LM, Huber M, Doores KJ, Falkowska E, Pejchal R, Julien JP, et al. Broad neutralization coverage of HIV by multiple highly potent antibodies. Nature (2011) 477:466-70. doi:10.1038/nature10373

35. Walker LM, Phogat SK, Chan-Hui PY, Wagner D, Phung P, Goss JL, et al. Broad and potent neutralizing antibodies from an African donor reveal a new HIV-1 vaccine target. Science (2009) 326:285-9. doi:10.1126/science.1178746

36. Mouquet H, Scharf L, Euler Z, Liu Y, Eden C, Scheid JF, et al. Complex-type $\mathrm{N}$-glycan recognition by potent broadly neutralizing HIV antibodies. Proc Natl Acad Sci USA (2012) 109:E3268-77. doi:10.1073/pnas.1217207109

37. Julien JP, Sok D, Khayat R, Lee JH, Doores KJ, Walker LM, et al. Broadly neutralizing antibody PGT121 allosterically modulates CD4 binding via recognition of the HIV-1 gp120 V3 base and multiple surrounding glycans. PLoS Pathog (2013) 9:e1003342. doi:10.1371/journal.ppat.1003342

38. Kong L, Lee JH, Doores KJ, Murin CD, Julien JP, McBride R, et al. Supersite of immune vulnerability on the glycosylated face of HIV-1 envelope glycoprotein gp120. Nat Struct Mol Biol (2013) 20:796-803. doi:10.1038/ nsmb. 2594

39. Doores KJ, Kong L, Krumm SA, Le KM, Sok D, Laserson U, et al. Two classes of broadly neutralizing antibodies within a single lineage directed to the high-mannose patch of HIV envelope. J Virol (2015) 89:1105-18. doi:10.1128/jvi02905-14

40. Sok D, Doores KJ, Briney B, Le KM, Saye-Francisco KL, Ramos A, et al. Promiscuous glycan site recognition by antibodies to the high-mannose patch of gp120 broadens neutralization of HIV. Sci Transl Med (2014) 6:236ra63. doi:10.1126/scitranslmed.3008104

41. Garces F, Sok D, Kong L, McBride R, Kim HJ, Saye-Francisco KF, et al. Structural evolution of glycan recognition by a family of potent HIV antibodies. Cell (2014) 159:69-79. doi:10.1016/j.cell.2014.09.009

42. Garces F, Lee JH, de Val N, de la Pena AT, Kong L, Puchades C, et al. Affinity maturation of a potent family of HIV antibodies is primarily focused on accommodating or avoiding glycans. Immunity (2015) 43:1053-63. doi:10.1016/j.immuni.2015.11.007

43. Barouch DH, Whitney JB, Moldt B, Klein F, Oliveira TY, Liu J, et al. Therapeutic efficacy of potent neutralizing HIV-1-specific monoclonal antibodies in SHIV-infected rhesus monkeys. Nature (2013) 503:224-8. doi:10.1038/nature12744
44. Caskey M, Schoofs T, Gruell H, Settler A, Karagounis T, Kreider EF, et al. Antibody 10-1074 suppresses viremia in HIV-1-infected individuals. Nat Med (2017) 23:185-91. doi:10.1038/nm4268

45. Gristick HB, von Boehmer L, West AP Jr, Schamber M, Gazumyan A, Golijanin J, et al. Natively glycosylated HIV-1 Env structure reveals new mode for antibody recognition of the CD4-binding site. Nat Struct Mol Biol (2016) 23:906-15. doi:10.1038/nsmb.3291

46. Morris CD, Azadnia P, de Val N, Vora N, Honda A, Giang E, et al. Differential antibody responses to conserved HIV-1 neutralizing epitopes in the context of multivalent scaffolds and native-like gp140 trimers. mBio (2017) 8:e36-17. doi:10.1128/mBio.00036-17

47. Kong L, He L, de Val N, Vora N, Morris CD, Azadnia P, et al. Uncleaved prefusion-optimized gp140 trimers derived from analysis of HIV-1 envelope metastability. Nat Commun (2016) 7:12040. doi:10.1038/ncomms12040

48. He L, de Val N, Morris CD, Vora N, Thinnes TC, Kong L, et al. Presenting native-like trimeric HIV-1 antigens with self-assembling nanoparticles. Nat Commun (2016) 7:12041. doi:10.1038/ncomms12041

49. Suloway C, Pulokas J, Fellmann D, Cheng A, Guerra F, Quispe J, et al. Automated molecular microscopy: the new Leginon system. J Struct Biol (2005) 151:41-60. doi:10.1016/j.jsb.2005.03.010

50. Lander GC, Stagg SM, Voss NR, Cheng A, Fellmann D, Pulokas J, et al. Appion: an integrated, database-driven pipeline to facilitate EM image processing. J Struct Biol (2009) 166:95-102. doi:10.1016/j.jsb.2009.01.002

51. Sorzano CO, Bilbao-Castro JR, Shkolnisky Y, Alcorlo M, Melero R, Caffarena-Fernández $G$, et al. A clustering approach to multireference alignment of single-particle projections in electron microscopy. J Struct Biol (2010) 171:197-206. doi:10.1016/j.jsb.2010.03.011

52. Tang G, Peng L, Baldwin PR, Mann DS, Jiang W, Rees I, et al. EMAN2: an extensible image processing suite for electron microscopy. J Struct Biol (2007) 157:38-46. doi:10.1016/j.jsb.2006.05.009

53. Pettersen EF, Goddard TD, Huang CC, Couch GS, Greenblatt DM, Meng EC, et al. UCSF chimera - A visualization system for exploratory research and analysis. J Comput Chem (2004) 25:1605-12. doi:10.1002/jcc.20084

54. Zhu Z, Dimitrov DS. Construction of a large naïve human phage-displayed Fab library through one-step cloning. Methods Mol Biol (2009) 525:129-42. doi:10.1007/978-1-59745-554-1_6

55. Kwong PD, Chuang GY, DeKosky BJ, Gindin T, Georgiev IS, Lemmin T, et al. Antibodyomics: bioinformatics technologies for understanding B-cell immunity to HIV-1. Immunol Rev (2017) 275:108-28. doi:10.1111/imr.12480

56. Altschul SF, Gish W, Miller W, Myers EW, Lipman DJ. Basic local alignment search tool. J Mol Biol (1990) 215:403-10. doi:10.1006/jmbi.1990.9999

57. Pearson WR, Lipman DJ. Improved tools for biological sequence comparison. Proc Natl Acad Sci USA (1988) 85:2444-8. doi:10.1073/pnas.85.8.2444

58. Larkin MA, Blackshields G, Brown NP, Chenna R, McGettigan PA, McWilliam $\mathrm{H}$, et al. Clustal W and clustal X version 2.0. Bioinformatics (2007) 23:2947-8. doi:10.1093/bioinformatics/btm404

59. Edgar RC. MUSCLE: multiple sequence alignment with high accuracy and high throughput. Nucleic Acids Res (2004) 32:1792-7. doi:10.1093/nar/gkh340

60. Li WZ, Godzik A. Cd-hit: a fast program for clustering and comparing large sets of protein or nucleotide sequences. Bioinformatics (2006) 22:1658-9. doi:10.1093/bioinformatics/btl158

61. Wyatt R, Sodroski J. The HIV-1 envelope glycoproteins: fusogens, antigens, and immunogens. Science (1998) 280:1884-8. doi:10.1126/ science. 280.5371 .1884

62. Khayat R, Lee JH, Julien JP, Cupo A, Klasse PJ, Sanders RW, et al. Structural characterization of cleaved, soluble HIV-1 envelope glycoprotein trimers. J Virol (2013) 87:9865-72. doi:10.1128/jvi.01222-13

63. Klasse PJ, Depetris RS, Pejchal R, Julien JP, Khayat R, Lee JH, et al. Influences on trimerization and aggregation of soluble, cleaved HIV-1 SOSIP envelope glycoprotein. J Virol (2013) 87:9873-85. doi:10.1128/jvi.01226-13

64. Ringe RP, Sanders RW, Yasmeen A, Kim HJ, Lee JH, Cupo A, et al. Cleavage strongly influences whether soluble HIV-1 envelope glycoprotein trimers adopt a native-like conformation. Proc Natl Acad Sci USA (2013) 110:1825661. doi:10.1073/pnas.1314351110

65. Sanders RW, Derking R, Cupo A, Julien JP, Yasmeen A, de Val N, et al. A next-generation cleaved, soluble HIV-1 Env trimer, BG505 SOSIP.664 gp140, expresses multiple epitopes for broadly neutralizing but not non-neutralizing antibodies. PLoS Pathog (2013) 9:e1003618. doi:10.1371/journal. ppat. 1003618 
66. Sanders RW, Moore JP. HIV: a stamp on the envelope. Nature (2014) 514:437-8. doi:10.1038/nature13926

67. Yasmeen A, Ringe R, Derking R, Cupo A, Julien JP, Burton DR, et al. Differential binding of neutralizing and non-neutralizing antibodies to native-like soluble HIV-1 Env trimers, uncleaved Env proteins, and monomeric subunits. Retrovirology (2014) 11:41. doi:10.1186/1742-4690-11-41

68. Derking R, Ozorowski G, Sliepen K, Yasmeen A, Cupo A, Torres JL, et al. Comprehensive antigenic map of a cleaved soluble HIV-1 envelope trimer. PLoS Pathog (2015) 11:e1004767. doi:10.1371/journal.ppat.1004767

69. Julien JP, Cupo A, Sok D, Stanfield RL, Lyumkis D, Deller MC, et al. Crystal structure of a soluble cleaved HIV-1 envelope trimer. Science (2013) 342:1477-83. doi:10.1126/science.1245625

70. Lyumkis D, Julien JP, de Val N, Cupo A, Potter CS, Klasse PJ, et al. Cryo-EM structure of a fully glycosylated soluble cleaved HIV-1 envelope trimer. Science (2013) 342:1484-90. doi:10.1126/science.1245627

71. Pancera M, Zhou T, Druz A, Georgiev IS, Soto C, Gorman J, et al. Structure and immune recognition of trimeric pre-fusion HIV-1 Env. Nature (2014) 514:455-61. doi:10.1038/nature13808

72. Kwon YD, Pancera M, Acharya P, Georgiev IS, Crooks ET, Gorman J, et al. Crystal structure, conformational fixation and entry-related interactions of mature ligand-free HIV-1 Env. Nat Struct Mol Biol (2015) 22:522-31. doi:10.1038/nsmb.3051

73. Stewart-Jones GB, Soto C, Lemmin T, Chuang GY, Druz A, Kong R, et al. Trimeric HIV-1-Env structures define glycan shields from clades A, B, and G. Cell (2016) 165:813-26. doi:10.1016/j.cell.2016.04.010

74. Lee JH, Ozorowski G, Ward AB. Cryo-EM structure of a native, fully glycosylated, cleaved HIV-1 envelope trimer. Science (2016) 351:1043-8. doi:10.1126/science.aad2450

75. Lee JH, Andrabi R, Su CY, Yasmeen A, Julien JP, Kong L, et al. A broadly neutralizing antibody targets the dynamic HIV envelope trimer apex via a long, rigidified, and anionic beta-hairpin structure. Immunity (2017) 46:690-702. doi:10.1016/j.immuni.2017.03.017

76. Guenaga J, Dubrovskaya V, de Val N, Sharma SK, Carrette B, Ward AB, et al. Structure-guided redesign increases the propensity of HIV Env to generate highly stable soluble trimers. J Virol (2015) 90:2806-17. doi:10.1128/ jvi.02652-15

77. Sharma SK, de Val N, Bale S, Guenaga J, Tran K, Feng Y, et al. Cleavageindependent HIV-1 Env trimers engineered as soluble native spike mimetics for vaccine design. Cell Rep (2015) 11:539-50. doi:10.1016/j.celrep. 2015.03.047

78. Guenaga J, Garces F, de Val N, Stanfield RL, Dubrovskaya V, Higgins B, et al. Glycine substitution at helix-to-coil transitions facilitates the structural determination of a stabilized subtype C HIV envelope glycoprotein. Immunity (2017) 46:792-803. doi:10.1016/j.immuni.2017.04.014

79. Georgiev IS, Joyce MG, Yang Y, Sastry M, Zhang B, Baxa U, et al. Singlechain soluble BG505.SOSIP gp140 trimers as structural and antigenic mimics of mature closed HIV-1 Env. J Virol (2015) 89:5318-29. doi:10.1128/ jvi.03451-14

80. Sok D, van Gils MJ, Pauthner M, Julien JP, Saye-Francisco KL, Hsueh J, et al. Recombinant HIV envelope trimer selects for quaternary-dependent antibodies targeting the trimer apex. Proc Natl Acad Sci USA (2014) 111:17624-9. doi:10.1073/pnas.1415789111

81. Wu X, Yang ZY, Li Y, Hogerkorp CM, Schief WR, Seaman MS, et al. Rational design of envelope identifies broadly neutralizing human monoclonal antibodies to HIV-1. Science (2010) 329:856-61. doi:10.1126/science.1187659

82. Falkowska E, Le KM, Ramos A, Doores KJ, Lee JH, Blattner C, et al. Broadly neutralizing HIV antibodies define a glycan-dependent epitope on the prefusion conformation of gp41 on cleaved envelope trimers. Immunity (2014) 40:657-68. doi:10.1016/j.immuni.2014.04.009

83. Huang J, Kang BH, Pancera M, Lee JH, Tong T, Feng Y, et al. Broad and potent HIV-1 neutralization by a human antibody that binds the gp41-gp120 interface. Nature (2014) 515:138-42. doi:10.1038/nature136

84. Doria-Rose NA, Bhiman JN, Roark RS, Schramm CA, Gorman J, Chuang GY, et al. New member of the V1V2-directed CAP256-VRC26 lineage that shows increased breadth and exceptional potency. J Virol (2016) 90:76-91. doi:10.1128/jvi.01791-15

85. Kong R, Xu K, Zhou T, Acharya P, Lemmin T, Liu K, et al. Fusion peptide of HIV-1 as a site of vulnerability to neutralizing antibody. Science (2016) 352:828-33. doi:10.1126/science.aae0474
86. Sanders RW, Moore JP. Native-like Env trimers as a platform for HIV-1 vaccine design. Immunol Rev (2017) 275:161-82. doi:10.1111/imr.12481

87. Ward AB, Wilson IA. The HIV-1 envelope glycoprotein structure: nailing down a moving target. Immunol Rev (2017) 275:21-32. doi:10.1111/ imr. 12507

88. Smith GP. Filamentous fusion phage - novel expression vectors that display cloned antigens on the virion surface. Science (1985) 228:1315-7. doi:10.1126/science.4001944

89. Huse WD, Sastry L, Iverson SA, Kang AS, Alting-Mees M, Burton DR, et al. Generation of a large combinatorial library of the immunoglobulin repertoire in phage lambda. Science (1989) 246:1275-81. doi:10.1126/science.2531466

90. Winter G, Griffiths AD, Hawkins RE, Hoogenboom HR. Making antibodies by phage display technology. Annu Rev Immunol (1994) 12:433-55. doi:10.1146/annurev.iy.12.040194.002245

91. Rader C, Barbas CF. Phage display of combinatorial antibody libraries. Curr Opin Biotechnol (1997) 8:503-8. doi:10.1016/s0958-1669(97)80075-4

92. Hoogenboom HR, de Bruïne AP, Hufton SE, Hoet RM, Arends JW, Roovers RC. Antibody phage display technology and its applications. Immunotechnology (1998) 4:1-20. doi:10.1016/s1380-2933(98)00007-4

93. Kretzschmar T, von Ruden T. Antibody discovery: phage display. Curr Opin Biotechnol (2002) 13:598-602. doi:10.1016/s0958-1669(02)00380-4

94. Bradbury ARM, Marks JD. Antibodies from phage antibody libraries. J Immunol Methods (2004) 290:29-49. doi:10.1016/j.jim.2004.04.007

95. Burton DR, Pyati J, Koduri R, Sharp SJ, Thornton GB, Parren PW, et al. Efficient neutralization of primary isolates of HIV-1 by a recombinant human monoclonal antibody. Science (1994) 266:1024-7. doi:10.1126/ science. 7973652

96. McCoy LE, Burton DR. Identification and specificity of broadly neutralizing antibodies against HIV. Immunol Rev (2017) 275:11-20. doi:10.1111/ imr.12484

97. Ravn U, Didelot G, Venet S, Ng KT, Gueneau F, Rousseau F, et al. Deep sequencing of phage display libraries to support antibody discovery. Methods (2013) 60:99-110. doi:10.1016/j.ymeth.2013.03.001

98. Xie J, Yea K, Zhang H, Moldt B, He L, Zhu J, et al. Prevention of cell death by antibodies selected from intracellular combinatorial libraries. Chem Biol (2014) 21:274-83. doi:10.1016/j.chembiol.2013.12.006

99. Hammers CM, Stanley JR. Antibody phage display: technique and applications. J Invest Dermatol (2014) 134(2):e17. doi:10.1038/jid.2013.521

100. Glanville J, D’Angelo S, Khan TA, Reddy ST, Naranjo L, Ferrara F, et al. Deep sequencing in library selection projects: what insight does it bring? Curr Opin Struct Biol (2015) 33:146-60. doi:10.1016/j.sbi.2015.09.001

101. Dai K, He L, Khan SN, O’Dell S, McKee K, Tran K, et al. Rhesus macaque $\mathrm{B}$-cell responses to an HIV-1 trimer vaccine revealed by unbiased longitudinal repertoire analysis. mBio (2015) 6:e1375-1315. doi:10.1128/mBio.01375-15

102. Hu JK, Crampton JC, Cupo A, Ketas T, van Gils MJ, Sliepen K, et al. Murine antibody responses to cleaved soluble HIV-1 envelope trimers are highly restricted in specificity. J Virol (2015) 89:10383-98. doi:10.1128/jvi.01653-15

103. Haynes BF, Mascola JR. The quest for an antibody-based HIV vaccine. Immunol Rev (2017) 275:5-10. doi:10.1111/imr.12517

104. DeKosky BJ, Ippolito GC, Deschner RP, Lavinder JJ, Wine Y, Rawlings BM, et al. High-throughput sequencing of the paired human immunoglobulin heavy and light chain repertoire. Nat Biotechnol (2013) 31:166-9. doi:10.1038/ nbt. 2492

105. DeKosky BJ, Kojima T, Rodin A, Charab W, Ippolito GC, Ellington AD, et al. In-depth determination and analysis of the human paired heavy- and lightchain antibody repertoire. Nat Med (2015) 21:86-91. doi:10.1038/nm.3743

Conflict of Interest Statement: The authors declare that the research was conducted in the absence of any commercial or financial relationships that could be construed as a potential conflict of interest.

Copyright $\odot 2017$ He, Lin, de Val, Saye-Francisco, Mann, Augst, Morris, Azadnia, Zhou, Sok, Ozorowski, Ward, Burton and Zhu. This is an open-access article distributed under the terms of the Creative Commons Attribution License (CC BY). The use, distribution or reproduction in other forums is permitted, provided the original author(s) or licensor are credited and that the original publication in this journal is cited, in accordance with accepted academic practice. No use, distribution or reproduction is permitted which does not comply with these terms. 